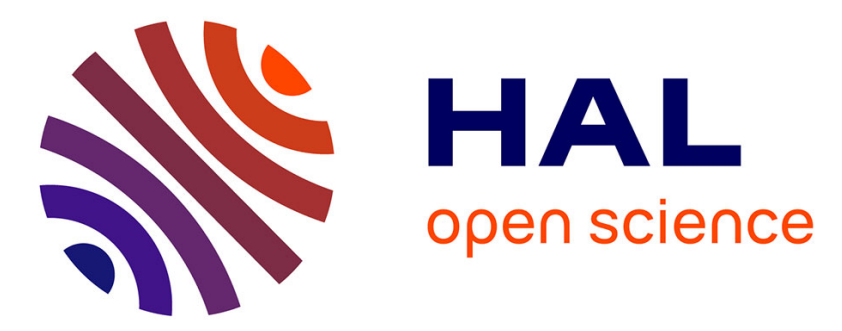

\title{
La modélisation par objets typés - une méthode de représentation pour les systèmes d'apprentissage et d'aide à la tâche - \\ Gilbert Paquette
}

\section{- To cite this version:}

Gilbert Paquette. La modélisation par objets typés - une méthode de représentation pour les systèmes d'apprentissage et d'aide à la tâche -. Sciences et Techniques Educatives, 1996, 3 (1), pp.9-42. edutice00135853

\section{HAL Id: edutice-00135853}

https://edutice.archives-ouvertes.fr/edutice-00135853

Submitted on 9 Mar 2007

HAL is a multi-disciplinary open access archive for the deposit and dissemination of scientific research documents, whether they are published or not. The documents may come from teaching and research institutions in France or abroad, or from public or private research centers.
L'archive ouverte pluridisciplinaire HAL, est destinée au dépôt et à la diffusion de documents scientifiques de niveau recherche, publiés ou non, émanant des établissements d'enseignement et de recherche français ou étrangers, des laboratoires publics ou privés. 


\title{
La modélisation par objets typés \\ - une méthode de représentation pour les systèmes d'apprentissage et d'aide à la tâche -
}

\author{
par Gilbert Paquette \\ Laboratoire LICEF, Télé-université \\ 1001 rue Sherbrooke est, \\ Montréal H2X 3M4 Canada \\ gilbert_paquette@teluq.uquebec.ca
}

\begin{abstract}
Résumé
Nous présentons une méthode de représentation des connaissances appelée "Modélisation par objets typés" (MOT) adaptée aux besoins de concepteurs pédagogiques qui désirent définir un système d'apprentissage ou un système d'aide à la tâche. Un formalisme graphique a été développé en visant un certain nombre d'objectifs : simplicité d'utilisation par des usagers non familiers avec la modélisation des connaissances, expressivité des représentations dans une variété de situations et de domaines de connaissances, transparence de la syntaxe par rapport aux relations entre les connaissances et à la sémantique d'un domaine. L'originalité de ce système de représentation réside dans le traitement intégré de quatre types de connaissances, les faits, les concepts, les procédures et les principes, et de six types de liens soumis à certaines contraintes d'intégrité ; elle tient également à sa capacité à représenter des catégories de modèles complexes tels que les systèmes conceptuels, les théories, les processus et les méthodes. Sur cette base, nous présentons également un éditeur graphique qui a fait l'objet d'un certain nombre d'expérimentations de terrain pour la conception pédagogique et la définition de systèmes éducatifs. Nous évaluons les résultats et identifions les perspectives pour des recherches ultérieures.
\end{abstract}

Mots-clés : modélisation pédagogique, formalismes de représentation, méthodes de représentation des connaissances, éditeur de modèles de connaissances.

\begin{abstract}
We report on a knowledge representation method called "Modeling using object types" (MOT) adapted to the needs of instructional designers who define learning systems and task support systems. A graphic formalism has been constucted aiming at a number of goals: simplicity of use by persons untrained in knowledge modeling techniques, representational expressiveness suited for a large variety of situations and knowledge domains, transparent view of relationships between knowledge units, uncovering a domain's semantic. The originality of the system lies in its capacity to represent, in an integrated way, four types of knowledge units, facts, concepts, procedures and principles, and six types of basic links between them obeing integrity constraints; it lies also in its capacity to represent complex model types such as conceptual systems, theories, processes and methods. Based on these basic constructs, we also present a computer assisted model builder which has been used in a certain number of field experiments for instructional design and education systems definition. We conclude by evaluating our results and identifying directions for future work.
\end{abstract}


Keywords: instructional modeling, representation formalism, knowledge representation methods, knowledge model editor. 


\section{Introduction}

Nous présentons ici une méthode de représentation appelée Modélisation par objets typés (MOT) adaptée aux besoins des concepteurs non-informaticiens qui désirent construire un système d'apprentissage ou un système d'aide à la tâche. Dans le cadre d'une pédagogie constructiviste, ces deux types de systèmes se rejoignent. Un système d'aide à la tâche, parce qu'il place l'usager en situation de résolution de problème et l'amène à construire de nouvelles connaissances. Inversement, un système d'apprentissage constructiviste peut utiliser un système d'aide à la tâche (ou de support à la performance) comme outil pour un ensemble d'activités d'apprentissage axés sur la résolution de problèmes.

Ce type de système est généralement constitué d'un certain nombre d'outils dont les relations doivent être précisées relativement à un ensemble de tâches à accomplir. Le concepteur a besoin d'un formalisme intégré, facile d'accès et capable de l'aider à construire une vue d'ensemble cohérente des principaux processus, concepts et stratégies qui décrivent une classe de problèmes à résoudre.

Il existe différents formalismes de représentation des connaissances tels les réseaux sémantiques, les schémas, les modèles entités-relations, les modèles de flux d'information, les modèles orientés objets. La plupart ont leur origine en intelligence artificielle et sont de plus en plus utilisés en génie logiciel. Citons à titre d'exemple les méthodes "Entités-relations" [Chen, 1976]; "Modern structured analysis" [Yourdon, 1989], "Object modeling technique (OMT)" [Rumbaugh et al, 1991], KADS [Scheiber et al, 1993]; graphes conceptuels [Sowa, 1984] pour n'en nommer que quelques unes.

Une des particularités du système de représentation présenté ici est le traitement intégré de deux niveaux d'abstraction, les faits et les connaissances abstraites, celles-ci étant à leur tour subdivisées en trois types : les concepts, les procédures et les principes. Habituellement, ces types de connaissances font l'objet de techniques de modélisation complémentaires mais différentes. Dans le système OMT par exemple, on spécifie un système au moyen de trois modèles (modèle objet, modèle dynamique, modèle fonctionnel), lesquels mettent en jeu de façon différente les faits, les concepts, les procédures et les principes de contrôle. Dans le système KADS, nous sommes en présence de sept modèles différents.

L'intégration de ces divers types de connaissances dans un même modèle est apparue nécessaire puisque, selon le type de connaissance, le traitement pédagogique sera différent. Par exemple, si la connaissance est un concept, on pourra la construire par un processus d'induction et de généralisation à l'aide d'exemples et de contre-exemples. Si la connaissance est une procédure ou un principe, on pourra la construire dans l'exercice de la résolution de problèmes de plus en plus complexe. On peut en dire autant de la conception des systèmes de support à la performance qui donnera lieu à des traitements différents selon que l'on a affaire à des faits (bases de données), des concepts (bases de connaissances, aide contextuelle), des procédures (outils de production) ou des principes (système experts et systèmes conseillers).

Les trois premières sections présentent le système de représentation MOT : la section 1 établit les bases théoriques du système de représentation ; la section 2 est consacrée à un premier tour d'horizon des composantes du système et à l'illustration de ses principes de représentation ; la section 3 présente le système MOT de façon formelle et en décrit les contraintes d'intégrité.

La méthode présentée ici est à l'origine de l'élaboration, au cours de l'année 1992, d'un enseignement universitaire sur le design pédagogique [Paquette, 1993] dont l'objectif était d'intégrer une forme de modélisation des connaissances aux activités d'un concepteur pédagogique. Puis, la méthode s'est raffinée et a finalement donné naissance à un outil modélisateur intégré à un Atelier de Génie didactique [Paquette et al, 1994] ${ }^{1}$. La section 4 présente brièvement l'outil modélisateur actuellement intégré à l'Atelier, mais dont l'utilité dépasse le strict domaine de la conception pédagogique. Enfin, la section 5 sera consacrée aux premiers résultats d'expérimentation et à l'énoncé des perspectives de développement.

\footnotetext{
${ }^{1}$ Cet article décrit le rôle de la modélisation des connaissances dans la conception pédagogique. Le modèle des connaissances décrivant le contenu, sert à définir les besoins et les objectifs de l'apprentissage. Il sert de base à la définition des stratégies et des scénarios d'apprentissage, ainsi que des instruments didactiques.
} 


\section{Bases théoriques de la modélisation par objets typés}

Nous présentons ici les bases théoriques du système de représentation MOT. Nous faisons d'abord appel au concept de schéma tel qu'il s'est développé en science cognitive, ce qui a conduit à l'identification de différents types de connaissances. Puis, nous présentons un certain nombre de travaux en sciences de l'éducation qui supposent également une identification des types de connaissances. Nous présentons ensuite un certain nombre de concepts liés à la collaboration et à la coopération entre agents, pour finalement énoncer les hypothèses à la base du système de représentation.

\subsection{Le concept de schéma et les types de connaissance}

Dans le système MOT, nous utilisons quatre types d'unités de connaissance : les faits, les concepts, les procédures et les principes. Des schémas seront utilisés pour décrire ces quatre types de connaissances de façon intégrée.

Le concept de schéma a marqué le passage du béhaviorisme au cognitivisme, comme approche théorique dominante en psychologie et dans les autres sciences cognitives. Dès les années vingt, Jean Piaget [Inhelder et Piaget, 1958] utilise les concepts de "schème", "schéma", "structure", "stratégie" et "opération" pour décrire les processus cognitifs. Selon Piaget, la croissance de l'intellect consiste essentiellement à développer des schémas de plus en plus logiques, nombreux et complexes. Parallèlement les psychologues "gestaltistes" comme [Wertheimer, 1945] développent les concepts voisins "d'entités", de "pattern" et de "structure". Egalement, le psychologue anglais [Bartlett, 1932], découvre que l'interprétation des textes semble guidée par des connaissances préexistantes qu'il décide d'appeler "schémas".

A la fin des années soixante, principalement aux Etats-Unis se développe la psychologie cognitive et l'intelligence artificielle telles que nous les connaissons aujourd'hui. [Bruner, 1973] contribue de façon substantielle à démontrer la validité, sur le plan psychologique, du concept de construction interne et de représentation des connaissances. [Newell et Simon, 1972] développent, sur cette même base, une représentation de l'activité de résolution de problèmes, pendant que [Minski, 1975] définit le concept de "frame" comme élément essentiel pour comprendre la perception, et aussi comme moyen de réconcilier la vision déclarative et la vision procédurale des connaissances [Winograd, 1985].

Misant sur la convergence de ces traditions de recherche, [Rumelhart et Ortony, 1977] ont défini la notion de schéma en y intégrant les points de vue suivants :

- le schéma comme structure de données mentales,

- le schéma pour représenter nos connaissances à propos des objets, des situations, des événements et des séquences d'actions [Anderson, 1985],

-le schéma en tant que scénario,

-le schéma en tant que théorie structurant les connaissances sur un sujet.

Les schémas jouent un rôle central dans la construction des connaissances. Ils guident la perception, définie comme un processus actif, constructif et sélectif; ils supportent la mémorisation et le souvenir qui consistent à créer ou à rechercher les schémas appropriés dans les réseaux sémantiques qui les associent; ils rendent la compréhension possible par la confrontation des schémas existants avec des événements nouveaux ; enfin, à travers les processus précédents, l'apprentissage est vu comme une transformation des schémas par les processus d'assimilation et d'accommodation. L'apprentissage est alors le résultat d'un processus de construction et de reconstruction des schémas, une interaction avec le monde physique et social plutôt qu'une simple recherche et un transfert des informations qu'il contient.

La distinction entre deux grandes catégories de schémas, les schémas déclaratifs ou conceptuels et les schémas procéduraux est maintenant traditionnelle. Les premiers structurent les données et les seconds contiennent les procédures ou méthodes de traitement pour organiser l'information. Plus récemment, une troisième grande catégorie de schémas appelés "conditionnels" [Paris, 1983] ou "stratégiques" a été proposée. Ceux-ci sont dotés d'une composante servant à déterminer le contexte et les conditions nécessaires au choix d'une séquence d'actions ou de procédures ou à la sélection d'un concept. 
Les schémas se prêtent bien à une représentation graphique des connaissances, qu'elle soit conceptuelle, procédurale ou stratégique. On identifie les principales composantes appelées attributs, ainsi que le type de "valeur" que ces attributs peuvent prendre. Ces valeurs sont des valeurs "concrètes" (un nombre, une couleur, une forme) ou d'autres schémas.

\subsection{Les types de connaissances en sciences de l'éducation}

De plus en plus, les travaux en sciences de l'éducation utilisent le concept de schéma tout en distinguant différents types de connaissances. L'intention derrière cette classification est de leur associer des stratégies pédagogiques correspondant aux connaissances visées par l'apprentissage.

Le "Component Display Theory (CDT)", développé par [Merrill, 1994] sur la base des travaux de Robert Gagné, suppose que les résultats de l'apprentissage peuvent être classifiés en un nombre limité de catégories. Cette théorie propose une classification mettant en jeu deux dimensions principales : la performance ${ }^{2}$ et le type de contenu. Il définit ainsi les "types de contenu":

- un fait est une association entre une date et un événement, ou un nom et une partie;

- un concept est un ensemble d'objets, d'événements ou de symboles qui partagent un certain nombre de caractéristiques communes;

- une procédure est un ensemble d'étapes permettant de conduire une action;

-un principe est une relation de cause à effet dans un processus." ${ }^{3}$

On retrouve ici les mêmes catégories de connaissances qui sont à la base du système de représentation MOT et auxquelles nous sommes arrivés également.

Dans un livre de référence très utilisé en design pédagogique, [Romiszowski, 1981] présente également une taxonomie des connaissances. Partant des performances attendues suite à un apprentissage, il distingue d'abord deux catégories d'habiletés : le souvenir et la compréhension qu'il associe respectivement à des connaissances factuelles et à des connaissances conceptuelles.

Il distingue ensuite les connaissances factuelles à propos d'objets, d'événements, de personnes et les connaissances factuelles sur comment procéder (les procédures). Puis, au niveau conceptuel, il distingue les concepts spécifiques permettant de reconnaître les instances d'un phénomène et de le définir et, d'autre part les règles ou principes qui permettent de relier les concepts et les faits de façon à pouvoir prédire ou expliquer des phénomènes.

Finalement, Romiszowski arrive lui aussi à quatre types de connaissances de base : les faits, les procédures, les concepts et les principes. Il mentionne que ce sont là les quatre catégories d'information utilisées par [Williams, 1977] pour étendre les taxonomies de Bloom et Gagné et qu'elles ressemblent également aux catégories d'information utilisées auparavant par [Horn 1969] comme base de ses "information mappings". 4

Un autre chercheur de pointe en éducation [Tennyson, 1988] propose un modèle de design pédagogique qui privilégie les connaissances contextuelles (ou stratégiques) dans les activités d'apprentissage. Décrivant les types de connaissances, Tennyson distingue les connaissances déclaratives, procédurales et contextuelles qu'il décrit ainsi:

-les connaissances déclaratives impliquent une attention à l'information du domaine et référent au "connaître que", par exemple, en soulignant les mots-clés dans un texte;

\footnotetext{
2 La "performance" de Merrill correspond à ce que Romiszowski appelle "habileté cognitive" et [Pitrat, 1990] métaconnaissance. Le système MOT présenté ici pourrait être étendu à ces métaconnaissances en ajoutant un nouveau type de connaissance les concernant et un nouveau lien "porte-sur" entre celles-ci et les autres types de connaissances. On pourrait traiter les objectifs d'apprentissage ainsi : "la (métaconnaissance de) mémorisation < porte-sur> la procédure de calcul de la surface d'un triangle rectangle".

3 Voir Merrill, p.109-112, traduction libre de l'auteur

${ }^{4}$ Romiszowski, p.241-243
} 
— les connaissances procédurales impliquent un "savoir comment" utiliser les concepts, règles et principes du domaine;

-les connaissances contextuelles impliquent "savoir quand et pourquoi" utiliser les concepts, règles et principes d'un domaine. ${ }^{5}$

D'autres auteurs ${ }^{6}$ tels que [West, 1991] rejoignent également ces trois catégories:

- les connaissances déclaratives peuvent être représentées en mémoire par un réseau associatif [Anderson, 1985] de propositions regroupant les faits et les concepts ; les réseaux pouvant être de nature sémantique ou épisodique ;

- les connaissances procédurales sont les savoir-faire se présentant sous la forme d'instruction à exécuter selon un certain ordre et à certaines conditions ;

- les connaissances conditionnelles consistent à savoir quand ou pourquoi utiliser une procédure ; elles ont la forme suivante : "si ces conditions sont respectées, alors faire telle et telle chose". 7

Bien que présentant certaines nuances avec les taxonomies de Merrill ou de Romiszowski, celles de Tennyson ou de West regroupent les faits et les concepts sous le vocable de "connaissances déclaratives", et les principes sous le vocable "connaissances contextuelles" ou "connaissances conditionnelles". On peut donc retenir de la littérature en sciences de l'éducation, quatre catégories d'unités de base : faits, concepts, procédures et principes.

\subsection{Agents, collaboration et coopération}

Les dimensions de collaboration ou de coopération entre les agents ${ }^{8}$ dans un processus ont été longuement négligées, bien qu'elles soient de toute première importance. Lorsqu'on modélise le contenu d'un système d'apprentissage, il faut pouvoir représenter cette coopération entre agents. Cette question doit également être prise en compte dans la définition des systèmes de support à la performance puisque, de plus en plus, les tâches se complexifiant, leur réalisation nécessite la collaboration et la coopération de plusieurs acteurs ou agents.

De plus en plus, les systèmes de représentation abordent ces questions, que ce soit dans le contexte de l'intelligence artificielle distribuée [Bond et Gasser 1988, Gasser 1991], les systèmes multi-agents cognitifs ou réactifs [Ferber, 1994] ou le génie logiciel. La méthodologie KADS [Schreiber et al, 1993] en génie logiciel propose un modèle de la coopération comme l'un de ses sept types de modèles :

-le modèle organisationnel, le modèle d'application et le modèle des tâches concernent ce qu'on pourrait appeler l'analyse préliminaire du problème : contexte organisationnel, problème à résoudre, tâches que le système doit pouvoir effectuer;

-le modèle de l'expertise et le modèle de la coopération sont les modèles centraux, définissant les principales composantes et le comportement du futur système ; le modèle conceptuel regroupe ces deux sous-modèles en un ensemble cohérent;

-le modèle de conception ("design model") s'intéresse aux choix des méthodes de représentation et de traitement nécessaire à l'implantation du modèle conceptuel.

Le modèle de l'expertise regroupe quatre niveaux de connaissances : objets du domaine ("domain knowledge"), méthodes d'inférence ("inference knowledge"), structure des tâches ("task knowledge") et stratégies ("strategic knowledge"). Ces connaissances correspondent aux quatre types de connaissances identifiées plus haut : le premier niveau concerne les faits et les concepts, le second niveau concerne les procédures de base, le troisième niveau complète la description des procédures et introduit certain principes de contrôle, alors que le quatrième niveau complète les connaissances stratégiques par l'adjonction de principes de contrôle de plus haut niveau.

\footnotetext{
5 Tennyson et Rasch, p. 372, traduction libre de l'auteur

${ }^{6}$ Voir aussi [Tardif, 1992]: "Pour un enseignement stratégique"

7 West, p. 15-16, traduction libre de l'auteur

${ }^{8}$ Dans un projet de Campus virtuel [Paquette et al, 1994] nous avons identifié les agents suivants: l'apprenant, le formateur, l'expert de contenu, le gestionnaire pédagogique et le concepteur.
} 
Le modèle de coopération consiste à spécifier les tâches qui nécessitent un effort de coopération et à les attribuer aux agents qui ont à coopérer dans le système. Il est basé sur trois éléments :

—une distribution des tâches qui assigne les sous-tâches à différents agents;

- la communication entre les agents qui assure que les dépendances entre tâches seront respectées, les extrants d'une tâche pouvant devenir intrants d'autres tâches;

-le contrôle, s'effectuant par la reconnaissance locale de l'agent apte à effectuer la tâche.

Dans le système de représentation MOT, ces divers éléments seront pris en compte en représentant les agents par des unités de connaissance de type concept, dont la composante principe tient compte de la fonction de contrôle spécifique à un agent, ainsi que celle de contrôle entre les agents. En liant ces unités de type principe avec les procédures qu'elles régissent par un lien spécial dit de réglementation, on effectue la distribution des tâches entre les agents. En décomposant le principe, on peut décrire la stratégie de communication et de contrôle à la base de la coopération avec autant de détail que nécessaire.

\subsection{Hypothèses et principes de base}

L'Hypothèse principale à la base du système de représentation MOT est qu'il est possible, à l'aide d'un petit nombre de types de connaissances et de liens entre ces connaissances, de représenter à la fois des modèles complexes tels que les systèmes conceptuels, les processus (incluant la coopération entre agents), les méthodologies et les théories.

Une hypothèse auxiliaire est qu'il est possible, dans une telle représentation basée sur un petit nombre de constituants primitifs, de construire des représentations relativement transparentes quant à leur sémantique.

Nous n'émettons toutefois aucune hypothèse sur l'efficacité de telles représentations dans les applications complexes de génie logiciel, mais seulement qu'elles s'avéreront efficace au niveau de l'organisation générale d'un système d'apprentissage ou d'un système de support à la performance.

Dans le but d'atteindre les objectifs qui précèdent, nous énonçons un certain nombre de principes que devra respecter le système de représentation et qui seront explicités dans la section suivante :

1. Toutes les connaissances seront représentées par des schémas dont la forme dépendra du type de connaissance : faits, concepts, procédures ou principes.

2. Toutes les relations entre connaissances qui ne sont pas des liens d'instanciation, de spécialisation, de composition, de précédence, d'intrant/extrant ou de réglementation (les 6 types de liens à la base du système), pourront être internalisées dans leur schéma d'origine, c'est-à-dire représentées en tant qu'attributs de ce schéma.

3. Toutes les connaissances abstraites (concepts, procédures, principes) pourront être instanciées pour produire un système de faits.

4. Toutes les connaissances abstraites (concepts, procédures, principes) pourront être organisées en hiérarchies.

5. Tout attribut d'un schéma, s'il est suffisamment complexe, pourra être externalisé sous la forme d'un nouveau schéma lié au premier par le lien de composition.

6. La notion de "processus" " pourra être représentée par une connaissance de type procédure munie de ses intrants et de ses extrants ; cette procédure principale pourra être décomposée en sous-

\footnotetext{
${ }^{9}$ Nous préférons le terme de processus à celui de tâche. On peut faire une distinction entre les deux, la tâche étant la description déclarative d'une production à effectuer, c'est-à-dire l'extrant du processus. Ce dernier a un sens plus dynamique, représentant tous les éléments de la démarche permettant d'effectuer une tâche ou un ensemble de tâches.
} 
procédures, liées ou non entre elles par des liens de précédence, et dotées de principes qui régissent le passage de contrôle d'une sous-procédure à une autre.

7. Les intrants ou données, ainsi que les extrants ou résultats d'une procédure pourront être représentés par des faits ou des concepts selon le niveau de généralité de la procédure.

8. La structure de contrôle d'une procédure pourra être externalisée sous forme de principes la régissant ou intégrée dans la procédure sous la forme d'une composante de type principe (règle de décision).

9. Les agents ou les acteurs d'un système distribué ou coopératif pourront être représentés sous la forme de schémas conceptuels mettant en évidence leurs composantes conceptuelles, procédurales ou stratégiques (leurs principes). On pourra se contenter de les représenter par des schémas de type principe si on ne désire mettre en évidence que leur aspect de contrôle.

\section{Types de connaissances et de liens}

Dans cette section, nous illustrons les principes qui viennent d'être énoncés, à l'aide d'exemple de schémas représentant les différents types de connaissances et de liens. Ce tour d'horizon nous permettra également de présenter le formalisme graphique utilisé pour construire les modèles par objets typés.

\subsection{Types de connaissances et schémas}

1. Toutes les connaissances pourront être représentées par des schémas dont la forme dépend du type de connaissance : faits, concepts, procédures ou principes.

2. Toutes les relations entre connaissances qui ne sont pas des liens d'instanciation, de spécialisation, de composition, de précédence, d'intrant/extrant ou de réglementation (les 6 types de liens à la base du système), pourront être internalisées dans leur schéma d'origine, c'est-à-dire représentés en tant qu'attributs de ce schéma.

Pour décrire un schéma, on identifie ses principales composantes ou "propriétés" appelées attributs, ainsi que le genre de "valeur" que ces attributs peuvent prendre. Selon que la connaissance est un concept, une procédure ou un principe, le schéma prendra une forme différente, ainsi qu'illustré sur la figure 1.

\begin{tabular}{|ll|}
\hline \multicolumn{2}{|c|}{ Triangle rectangle } \\
\hline \hline Type figure: $\quad$ Triangle \\
Nb angles: $\quad 3$ \\
Liste des angles: & $(\mathrm{A}, \mathrm{B}, \mathrm{C})$ \\
Nb. côtés: $\quad 3$ \\
Liste des côtés: $\quad$ (a, b, c) \\
Contrainte: $\quad$ Est-rectangle \\
Surface: $\quad$ Calculer surface \\
\hline
\end{tabular}

Concept

\begin{tabular}{|c|}
\hline \multicolumn{1}{|c|}{ Calculer surface } \\
\hline \hline Action 1: $\quad$ Obtenir A, B,C \\
Action 2: $\quad$ Obtenir a,b,c \\
Action 3: $\quad$ Vérifier si \\
"Est-rectangle" \\
Décision 1: $\quad$ Si oui, retourner \\
$\mathrm{a}^{* \mathrm{~b} / 2}$ dans résultat \\
Décision 2: Terminer \\
\hline
\end{tabular}

Procédure

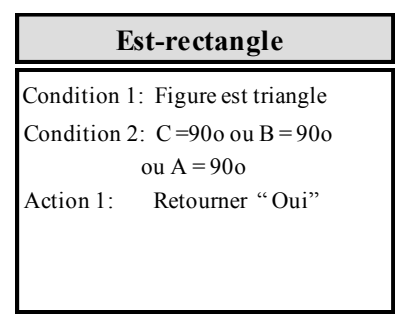

Principe

Figure 1 - Trois types de schémas

Les attributs d'un schéma de concept comme celui de "Triangle rectangle" ont pour valeur d'autres schémas de concepts, ou des schéma de procédure ou de principe.

-Les schémas de concept seront décrits de la même façon jusqu'à ce qu'on identifie des schémas de concept "simples" c'est-à-dire composés d'une seule propriété. C'est le cas ici de l'attribut "Nb 
angles" qui prend une valeur simple : "3", alors que "Type de figure" prend pour valeur un autre schéma de concept complexe, celui de "Triangle".

-Les schémas de procédure permettent de "calculer" la valeur d'un attribut, plutôt que de la spécifier directement. C'est le cas ici de l'attribut "Surface" dont la valeur sera le résultat de la procédure "Calculer surface".

-Les schémas de principe posent des "contraintes d'intégrité" que doivent respecter certains des attributs du concept. C'est le cas ici de l'attribut "Contrainte" qui a pour valeur le principe "Estrectangle" permettant de déterminer si le triangle est rectangle.

Les attributs d'un schéma de procédure sont les différentes "actions" qui composent la procédure. Ces actions peuvent être d'autres procédures (sous-procédures) comme "Vérifier si Est-rectangle" ou des décisions comme "Si oui, retourner $\mathrm{a}$ *b/2 dans résultat". Lorsqu'une action est une autre procédure, elle sera représentée de la même façon jusqu'à ce qu'on obtienne une procédure simple (une opération) telle que "Obtenir a,b,c". Par contre "Vérifier si Est-rectangle" est une procédure plus complexe qui pourrait à son tour être décrite par ses propres attributs.

Les attributs d'un schéma de principe sont soit d'autres principes (sous-principes), soit des conditions d'application ou des actions à effectuer si les conditions sont remplies. Ces actions sont représentées par des schémas de procédure alors que les sous-principes ou les conditions d'application sont représentés à leur tour par des schémas de principe jusqu'à ce qu'on obtienne des énoncés de principes simples comme $\mathrm{C}=90 \mathrm{o}$. Par contre "Est-rectangle" est un principe complexe qui est décomposé en deux conditions et une action.

Dans les schémas de la figure 1, on a internalisé certains liens entre connaissances. Par exemple, un lien externe est implicitement établi entre un triangle rectangle et la procédure permettant de calculer sa surface. Egalement, d'autres liens sont internalisés sous forme d'attribut d'un schéma. Par exemple le lien entre les concepts "Triangle" et "Triangle rectangle" devient un attribut de ce dernier concept. Nous verrons plus loin comment on peut externaliser un tel lien pour mettre en évidence la relation de spécialisation entre les deux concepts. Egalement un lien comme "a comme nombre de côtés" entre une figure géométrique et un nombre a aussi été internalisé. Contrairement à d'autres systèmes de représentation, un tel lien très particulier à un domaine de connaissance (ici la géométrie) ne sera pas externalisé dans le système MOT et devra être représenté comme attribut interne.

\subsection{Faits et lien d'instanciation (I)}

3. Toutes les connaissances abstraites (concepts, procédures, principes) pourront être instanciées pour produire un système de faits.

Un schéma, qu'il représente un concept, une procédure ou principe, agit comme un moule permettant de créer des exemples ou "instances", obtenues en donnant des valeurs précises aux attributs parmi toutes les valeurs possibles. Chaque instance et le schéma dont elle est issue sont liés par un lien d'instanciation "est-instancede" (I).

La figure 2 illustre ce fait en instanciant les schémas de la figure 1. Chacun des schémas peut donner lieu à plusieurs instances. Dans le cas du concept de Triangle rectangle, il s'agit de tous les triplets d'angles et de côtés respectant la contrainte "Est-rectangle". Pour obtenir une instance d'un concept comme celui de la figure 2, il faut spécifier ou obtenir des valeurs pour chacun des attributs qui n'a pas déjà une valeur précise. Dans ce cas-ci, il faut non seulement donner des valeurs aux angles et aux côtés, mais également instancier la contrainte et la procédure de calcul avec ces valeurs.

L'instanciation d'une procédure telle que "Calculer surface" est la trace obtenue en spécifiant l'un des cas possibles pour les valeurs de A,B,C et a,b,c. L'instanciation du principe "Est-rectangle" est l'énoncé particulier obtenu en spécifiant les valeurs : "Si la figure est un triangle et si $30 \mathrm{o}=90 \mathrm{o}$ ou $90 \mathrm{o}=60 \mathrm{o}$ ou $90 \mathrm{o}=90 \mathrm{o}$, alors retourner "oui". 


\begin{tabular}{|c|}
\hline Triangle rectangle \\
\hline 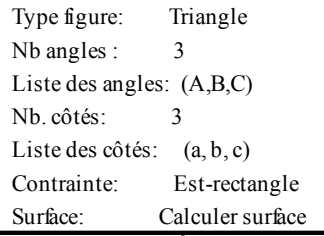 \\
\hline Exemple de triangle rectangle \\
\hline $\begin{array}{l}\text { Type figure: } \quad \text { Triangle } \\
\text { Nb angles : } \quad 3 \\
\text { Liste des angles: }(30 \mathrm{o}, 60 \mathrm{o}, 90 \mathrm{o}) \\
\text { Nb. côtés: } \quad 3 \\
\text { Liste des côtés: } \quad(3,4,5) \\
\text { Contrainte: } \quad \text { Oui } \\
\text { Surface: } \quad 6\end{array}$ \\
\hline
\end{tabular}

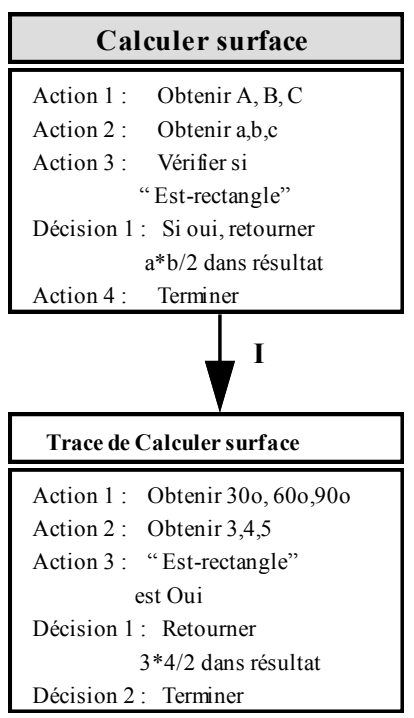

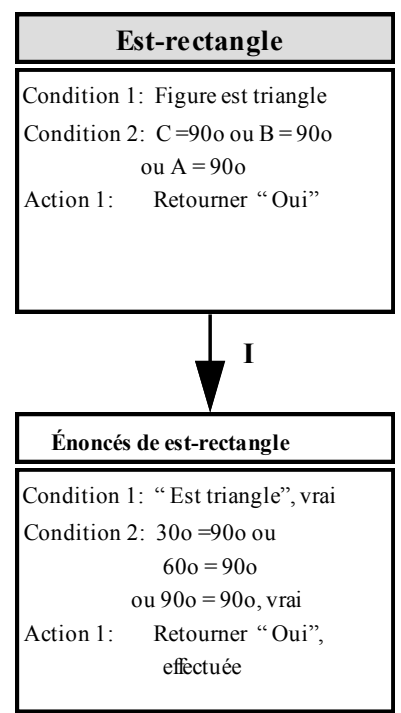

Figure 2 - Instanciation de schéma de concept, de procédure ou de principe

En fait, l'instanciation produit un nouveau schéma liant plusieurs faits entre eux, c'est-à-dire un système de faits tel le suivant :

-La figure est un triangle (déjà instancié dans le concept)

-La figure a trois angles

-La figure a pour angles $30 \mathrm{o}, 60 \mathrm{o}$ et $90 \mathrm{o}$

-La figure a trois côtés

-La figure a pour côtés 3,4 et 5

-La contrainte "Est-rectangle" est satisfaite

-La surface est de 6

De même, l'instanciation d'une procédure ou d'un principe produit un système de faits. Dans le cas de la procédure, on produit les faits suivants, liés entre eux par leur exécution temporelle :

-On a obtenu les angles $30 \mathrm{o}, 60 \mathrm{o}$ et $90 \mathrm{o}$

-On a obtenu les côtés 3,4 et 5

-On a vérifié "Est rectangle" et on a obtenu la valeur vrai

-On a décidé de faire le calcul et obtenu 6 comme résultat

-On a terminé la procédure.

Dans le cas d'un principe, l'instanciation consiste à remplacer les variables par des valeurs. On obtient pour la condition des énoncés factuels qui peuvent être vrais ou faux. S'il sont vrais, les actions sont effectuées, sinon, elles ne sont pas effectuées. Dans l'exemple :

-"Est-triangle" est vrai

$-30 \mathrm{o}=90 \mathrm{o}$ ou $60 \mathrm{o}=90 \mathrm{o}$ ou $90 \mathrm{o}=90 \mathrm{o}$ est vrai

- L'action "Retourner "Est-rectangle vrai" est effectuée 


\subsection{Hiérarchies et lien de spécialisation (S)}

4. Toutes les connaissances abstraites, concepts, procédures ou principes, pourront être organisées en hiérarchies.

Un schéma peut également être vu comme représentant une classe d'objets qui en sont les instances. Cette classe peut avoir des sous-classes obtenues, notamment en fixant un attribut à une valeur partagée par tous les membres de la sous-classe.

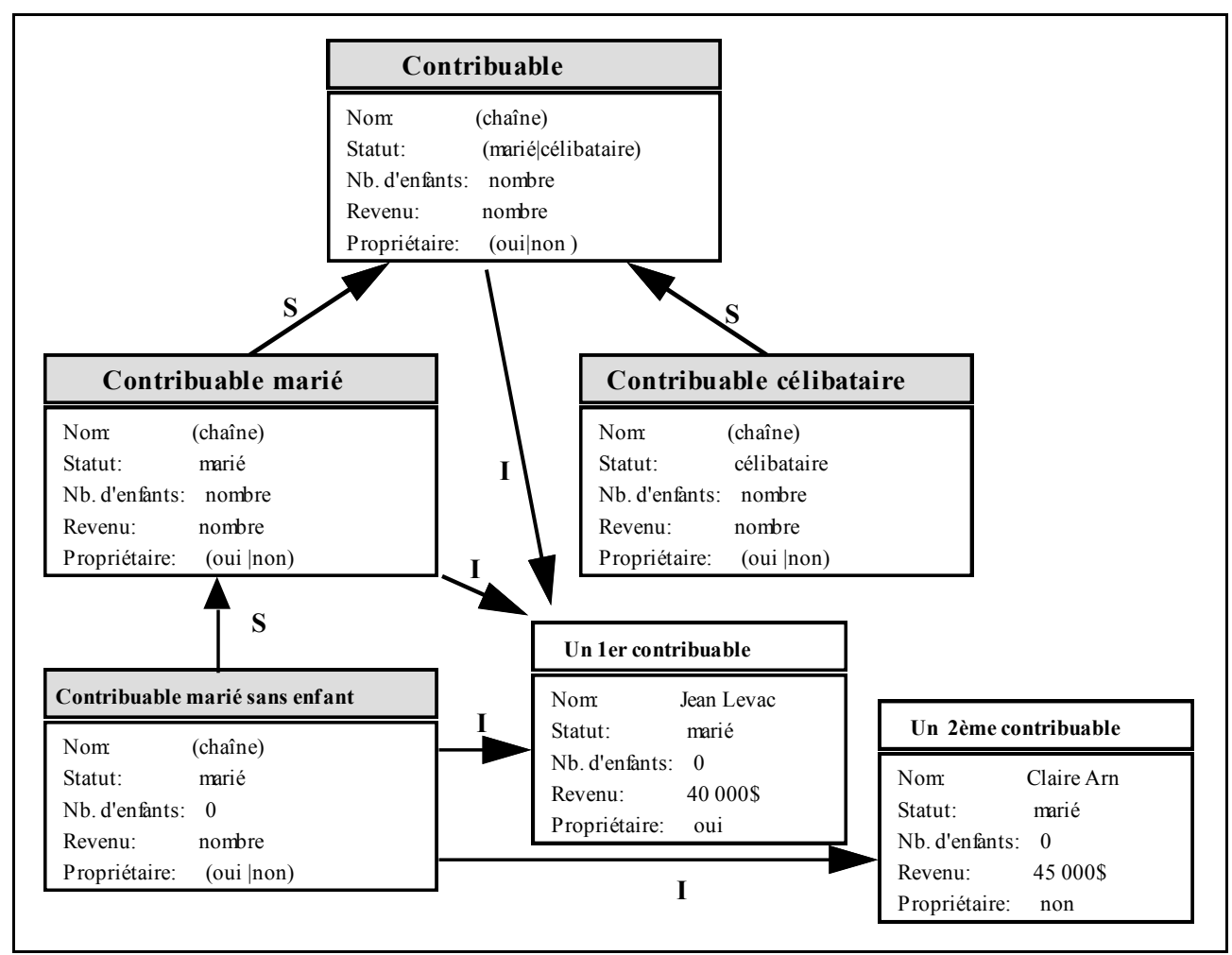

Figure 3 - Hiérarchie de schémas de concept

La figure 3 illustre ce fait. En fixant la valeur de l'attribut "Statut" à "marié" dans le schéma décrivant un contribuable, on obtient la sous-classe des contribuables mariés. Un Contribuable marié est une sorte de Contribuable, le premier schéma étant lié au second par un lien de spécialisation "est-une-sorte-de" (S); de même pour un contribuable célibataire.

On peut encore spécifier davantage le schéma pour obtenir la sous-classe des contribuables mariés n'ayant pas d'enfant. A la limite, si on spécifie tous les attributs, on obtient une instance, c'est-à-dire un système de faits. On peut alors convenir que ce lien, au plus bas niveau de la hiérarchie, est un lien d'instanciation. Même si un tel système de fait est instance de tous les schémas de la hiérarchie, on peut se contenter de spécifier un seul des liens d'instanciation I, celui au plus bas niveau.

On peut également construire des hiérarchies de procédures ou des hiérarchies de principes. Par exemple, une procédure de calcul de la surface d'un triangle rectangle est un cas particulier de la procédure de calcul d'un triangle quelconque. 


\subsection{Externalisation et liens de composition (C)}

5. Tout attribut d'un schéma, s'il est suffisamment complexe, pourra être externalisé sous la forme d'un nouveau schéma lié au premier par le lien de composition.

Au paragraphe 2.1, nous avons mis en évidence l'internalisation des liens dans les schémas en termes d'attribut. On pourra également faire l'opération inverse, c'est-à-dire externaliser les attributs sous la forme de schémas. On dira alors que ceux-ci sont des composantes du schéma d'origine et on tracera un lien "se compose de" $(\mathrm{C})$ entre ce dernier et ses composantes.

L'exemple de la figure 4 illustre ce fait. Il présente également une notation graphique abrégée qui ne précise pas nécessairement les liens internalisés sous forme d'attribut, mais uniquement les liens entre schémas. Pour mieux repérer les types de connaissances, les concepts sont présentés sous la forme de rectangles, les procédures sous la forme d'ovales et les principes sous la forme d'hexagones. Les instances (systèmes de faits) sont présentées par des boîtes de même forme, mais sans couleur de remplissage.

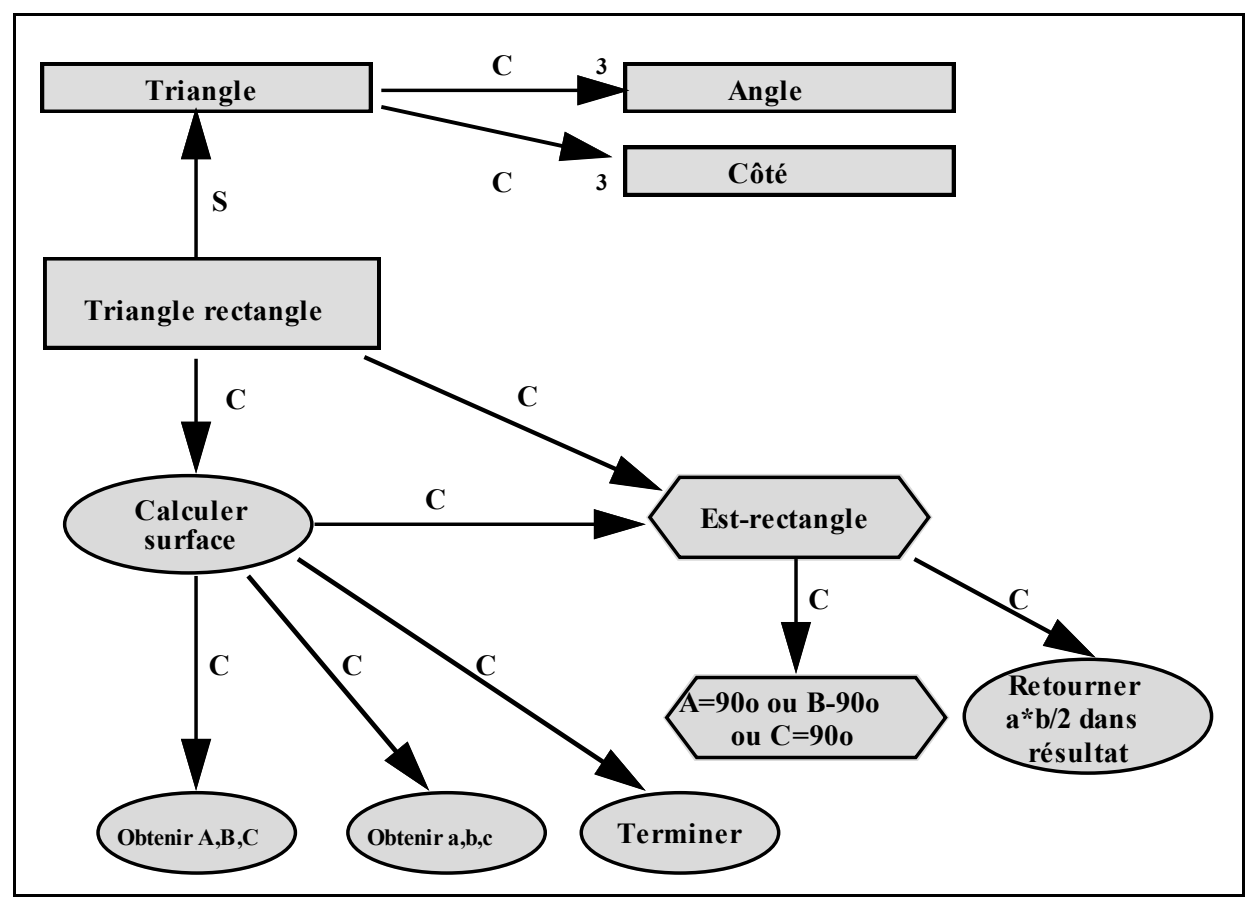

Figure 4 - Liens de composition entre schémas de concept, de procédure ou de principe

Par rapport à la figure 1, on peut constater que l'externalisation des principaux attributs sous forme de schémas liés par des liens $\mathrm{C}$, fait davantage ressortir la structure des connaissances d'un domaine et donc, du point de vue de l'usager, ce qu'il y a à apprendre ou à utiliser. Par ailleurs la procédure n'est pas complètement spécifiée quant à l'ordonnancement des actions. Cette question sera réglée par l'ajout au système de nouveaux types de liens. On note également la possibilité d'ajouter des indices de cardinalité sur les liens $\mathrm{C}$; cette précision est évidemment facultative. 


\subsection{Processus, liens de précédence (P) et d'intrant/extrant (I/E)}

6. La notion de "processus" pourra être représentée par une connaissance de type procédure munie de ses intrants et de ses extrants; cette procédure principale pourra être décomposée en sous-procédures, liées ou non entre elles par des liens de précédence, et dotées de principes qui régissent le passage de contrôle d'une sous-procédure à l'autre.

7. Les intrants ou données, ainsi que les extrants ou résultats d'une procédure pourront être représentés par des faits ou des concepts selon le niveau de généralité de la procédure.

Les connaissances procédurales sont celles qui nous permettent d'effectuer un ensemble de tâches et d'opérations reliées entre elles de façon plus ou moins complexe. La figure suivante met en évidence la nécessité de prévoir deux autres types de liens qui permettent de traiter la question de précédence (lien P) entre les sous-procédures ou entre une procédure et les données qui lui servent d'intrant ou qui sont des extrants de la procédure (lien I/E).

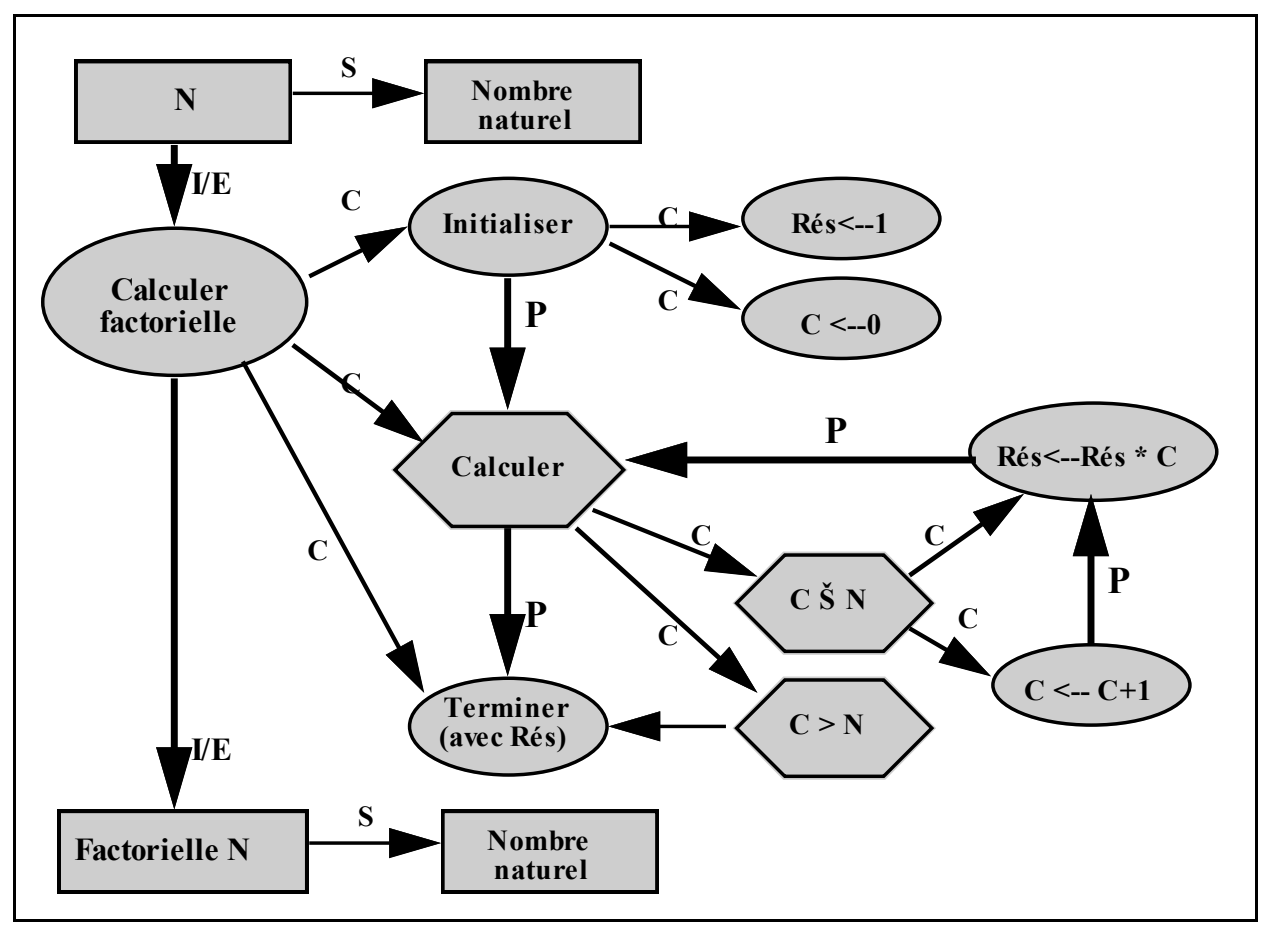

Figure 5 - Liens de précédence et d'intrant-extrant pour décrire une procédure

La figure 5 illustre l'usage de ces liens pour le calcul de factorielle $\mathrm{N}$ (c'est-à-dire 1 x 2 x 3 x... x N).

- La procédure "Calculer factorielle" a pour intrant (lien I/E) ${ }^{10}$ un concept $\mathrm{N}$ et pour extrant un concept Factorielle-N, lesquels sont deux nombres naturels. Cette description de la procédure au plus haut niveau indique déjà son degré de généralité. On pourra faire n'importe quel calcul de factorielle, il suffira d'instancier $\mathrm{N}$ à une valeur précise qui est un nombre naturel (entier positif ou nul).

- La procédure se décompose en une séquence de trois actions liées par des liens de précédence (P) : Initialiser, puis Calculer, puis Terminer. Dans certaines applications, une telle description de haut

\footnotetext{
10 Nous aurions pu prévoir ici deux liens au lieu d'un seul, soit un lien "Intrant” et un lien “Extrant”. Toutefois, cela n'est pas nécessaire puisque le contexte permet toujours d'interpréter correctement le lien.
} 
niveau suffira. Ici, on a voulu aller jusqu'aux opérations pour montrer que le formalisme a un pouvoir d'expression au moins aussi grand que d'autres modes de représentation des procédures.

- La première sous-procédure se décompose à son tour en deux opérations simples. Il faut noter que, contrairement à certains formalismes de représentation, il n'y a pas de lien de précédence entre ces deux opérations puisqu'elles pourraient très bien être effectuées en parallèle par deux agents différents.

- La seconde action est un principe composé de deux autres principes qui permettent de prendre une décision selon que la valeur de $\mathrm{C}$ a dépassé la valeur de $\mathrm{N}$ ou non. Dans le premier cas, le principe prend action et appelle la sous-procédure "Terminer", laquelle consiste essentiellement à retourner le résultat contenu dans la variable "Rés".

- L'autre principe, si sa condition est satisfaite (C n'a pas dépassé $N$ ), entreprend une autre itération en augmentant $C$ de 1 et effectuant une autre multiplication (Rés $* C$ ). Notons ici que l'ordre de ces opérations est vital, d'où l'usage des liens $\mathrm{P}$. Il en est de même du lien $\mathrm{P}$ qui part de la deuxième opération et qui permet de boucler en retournant à l'action "Calculer".

\subsection{Agents, contrôle et lien de réglementation ( $R$ )}

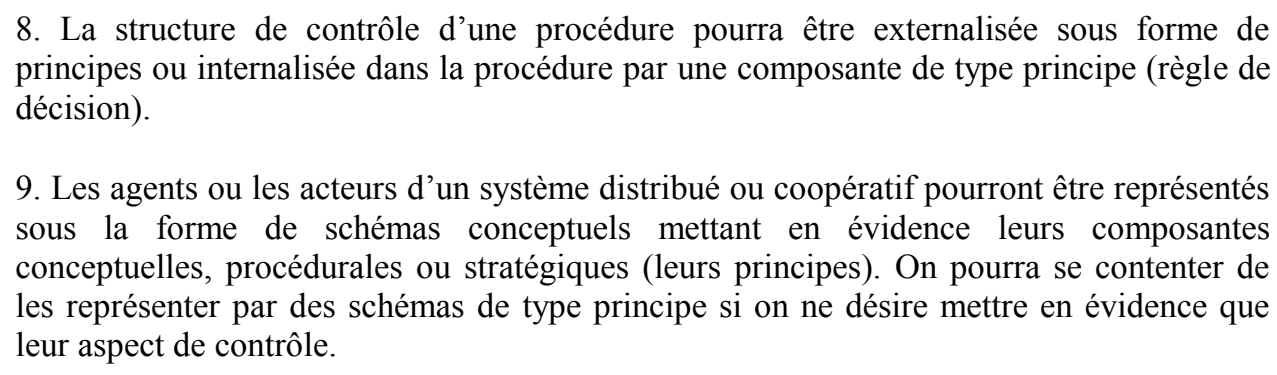

8. La structure de contrôle d'une procédure pourra être externalisée sous forme de principes ou internalisée dans la procédure par une composante de type principe (règle de décision).

9. Les agents ou les acteurs d'un système distribué ou coopératif pourront être représentés sous la forme de schémas conceptuels mettant en évidence leurs composantes conceptuelles, procédurales ou stratégiques (leurs principes). On pourra se contenter de les représenter par des schémas de type principe si on ne désire mettre en évidence que leur aspect de contrôle.

Les principes ou connaissances stratégiques sont celles qui nous permettent se savoir quand ou pourquoi appliquer nos autres connaissances. L'exemple de la figure 5 montre l'usage des principes comme composante de contrôle au sein de la procédure et de ses sous-procédures. Mais on peut aussi externaliser les connaissances de contrôle comme dans la figure 6 en les faisant régir une procédure de l'extérieur. Pour ce faire on utilise un sixième et dernier lien, le lien de réglementation $(\mathrm{R})$. 


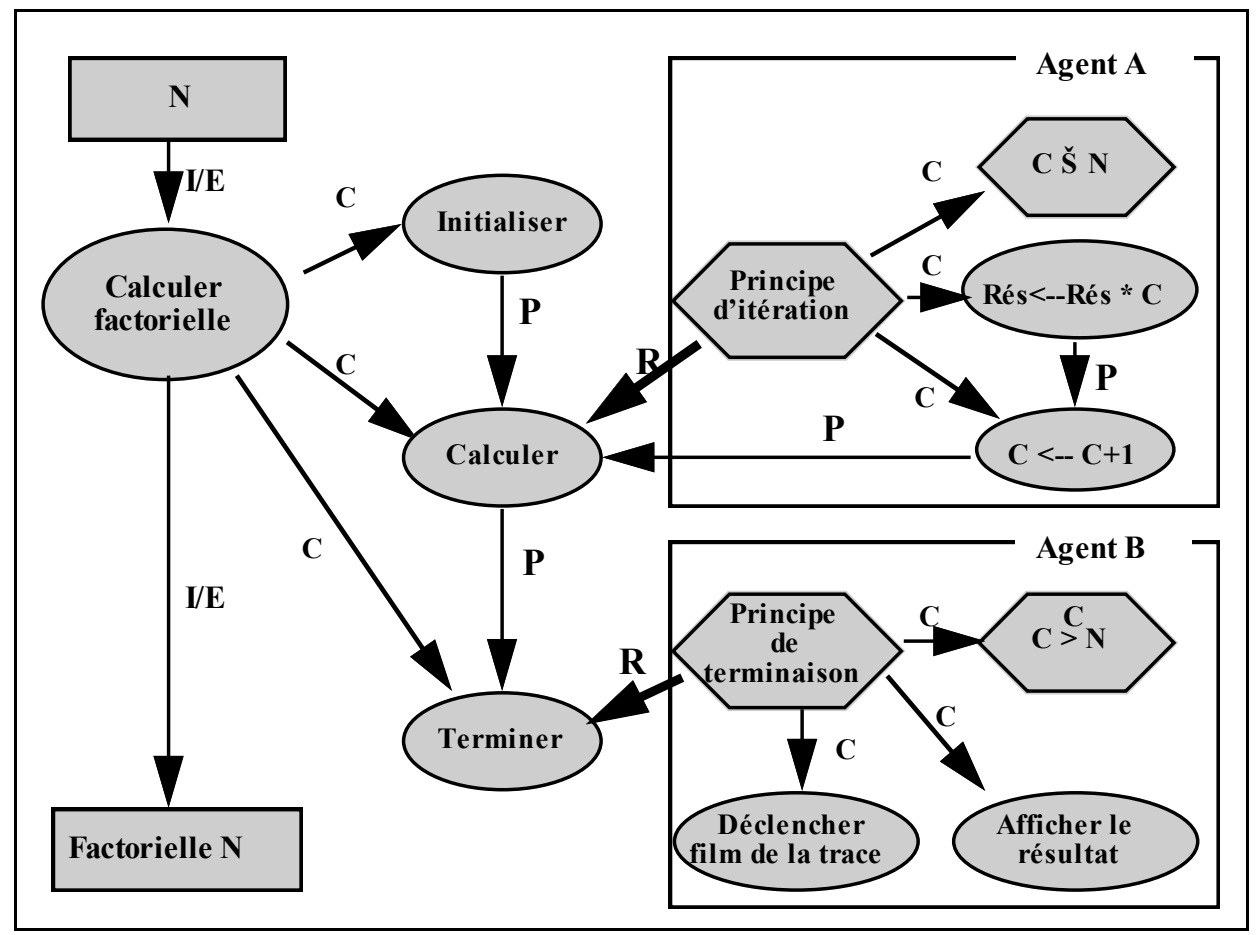

Figure 6- Liens de réglementation d'une procédure par des principes

La figure 6 présente une façon alternative de décrire l'algorithme de calcul de Factorielle N. La procédure n'est décrite qu'au niveau supérieur en spécifiant ses trois sous-procédures, son intrant et son extrant. Deux principes viennent de l'extérieur régir le contrôle entre les deux sous-procédures "Calculer" et "Terminer". Le premier principe effectue les calculs et gère les itérations. Lorsque sa condition n'est pas satisfaite, il passe le contrôle à la sous-procédure "Terminer". Là, un deuxième principe s'occupe de la présentation des résultats.

Les deux principes peuvent être gérés par un seul agent, compte tenu de la simplicité des tâches. Cependant, on peut aussi imaginer que ces tâches sont gérées par deux agents différents qui coopèrent dans l'accomplissement de la tâche ; le premier s'occupant des calculs et le second de la présentation des résultats. L'avantage du formalisme graphique est d'indiquer clairement la nature de leur coopération relativement aux tâches et aux sous-tâches à effectuer et leur participation respective au contrôle de la procédure.

La figure suivante apporte un autre exemple de la coopération entre agents dans le cas d'une tâche collaborative de rédaction d'un texte. On y retrouve un agent coordonateur qui régit les tâches d'élaboration du plan et de répartition des tâches, un ou des agents rédacteurs et un agent éditeur qui met en forme et imprime le document produit. 


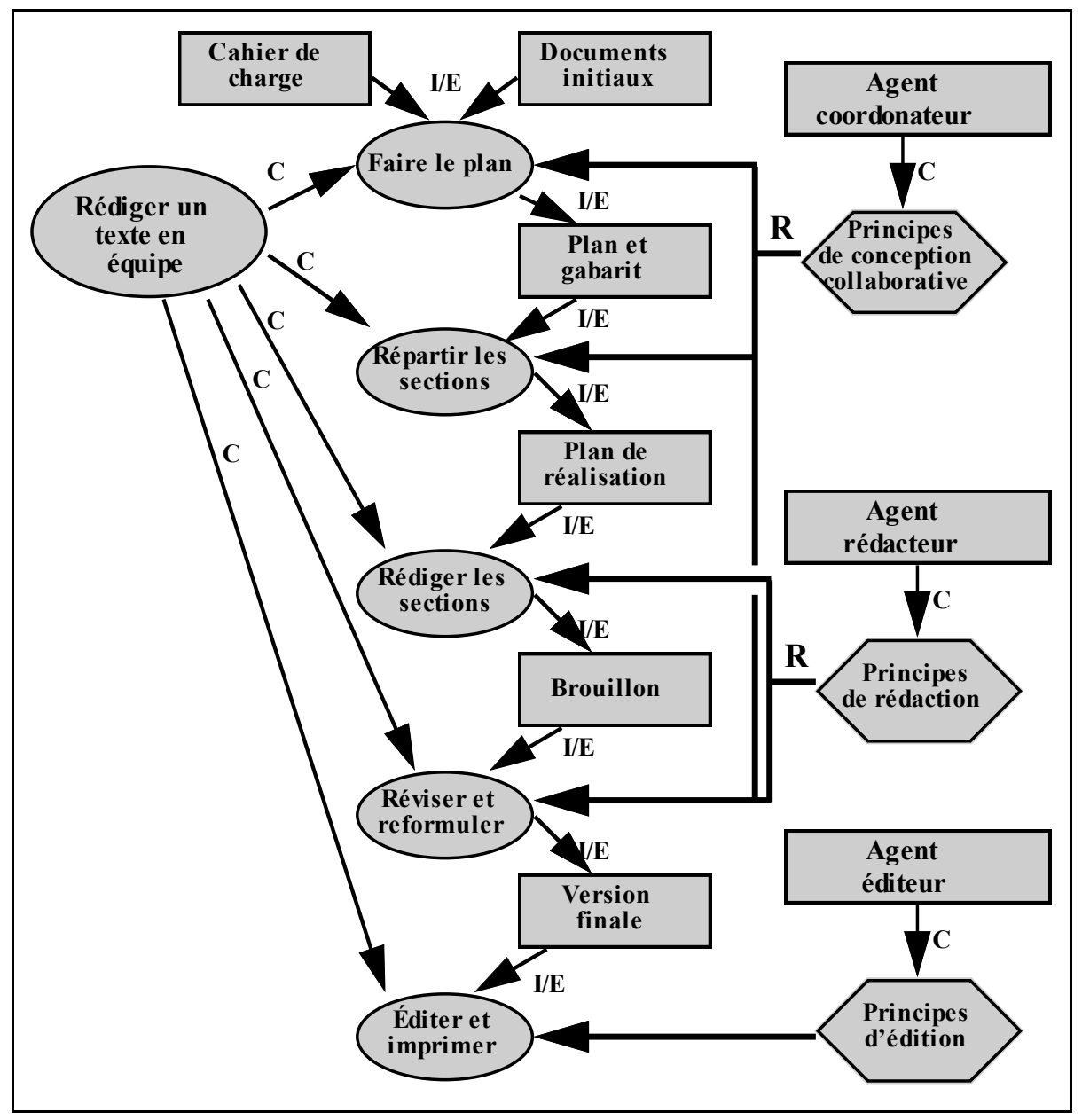

Figure 7 - Un processus d'écriture régit par trois types d'agents

Sur cette figure, nous avons représenté les agents par des concepts, ce qui permettrait de décrire leurs savoirs et leur savoir faire. Par exemple, l'agent coordonateur pourrait être décrit plus précisément par des composantes concept donnant son nom, sa formation et son expérience ou encore des composantes procédurales telles que des procédures de gestion.

Sur la figure, on a présenté explicitement la composante stratégique de ces agents, c'est-à-dire les principes de conception coopérative qui lui permettent de contrôler les tâches de rédaction d'un plan, de répartition du travail, de révision et de reformulation finale. Parmi les principes de conception, on pourrait retrouver notamment un principe comme "Parcourir les documents initiaux et intégrer les idées au fur et à mesure" ou "Réorganiser fréquemment les têtes de section tout en les décomposant", ou encore, "Réviser le plan une dernière fois en équipe avant de répartir les tâches".

\subsection{Systèmes de connaissances complexes}

Les outils de représentation qui précèdent permettent de modéliser des systèmes de connaissances plus complexes que les quelques exemples présentés. En général, les trois types de connaissances abstraites, concepts, procédures et principes, vont se combiner en des systèmes plus ou moins complexes de connaissances structurées. 
La figure 8 illustre cette idée. On y définit quatre types de systèmes composés : les systèmes conceptuels, les théories, les processus et les méthodes.

Les systèmes conceptuels sont des systèmes de connaissances constitués d'un nombre plus ou moins grand de concepts mis en relations sous la forme d'un réseau sémantique. Chaque concept est généralement un schéma conceptuel dont les attributs peuvent être d'autres schémas conceptuels, des procédures ou des principes.

Exemple : le domaine des véhicules automobiles comporte un concept "Automobile", qui est une sorte de "Véhicule automobile" tout comme le camion (lien S). Le concept "Automobile" est décrit par des concepts le composant (liens C) tels que celui de moteur ou celui de transmission. Le concept "Transmission" a notamment comme composante une "Procédure d'entretien", laquelle contient à son tour des "Règles d'entretien".

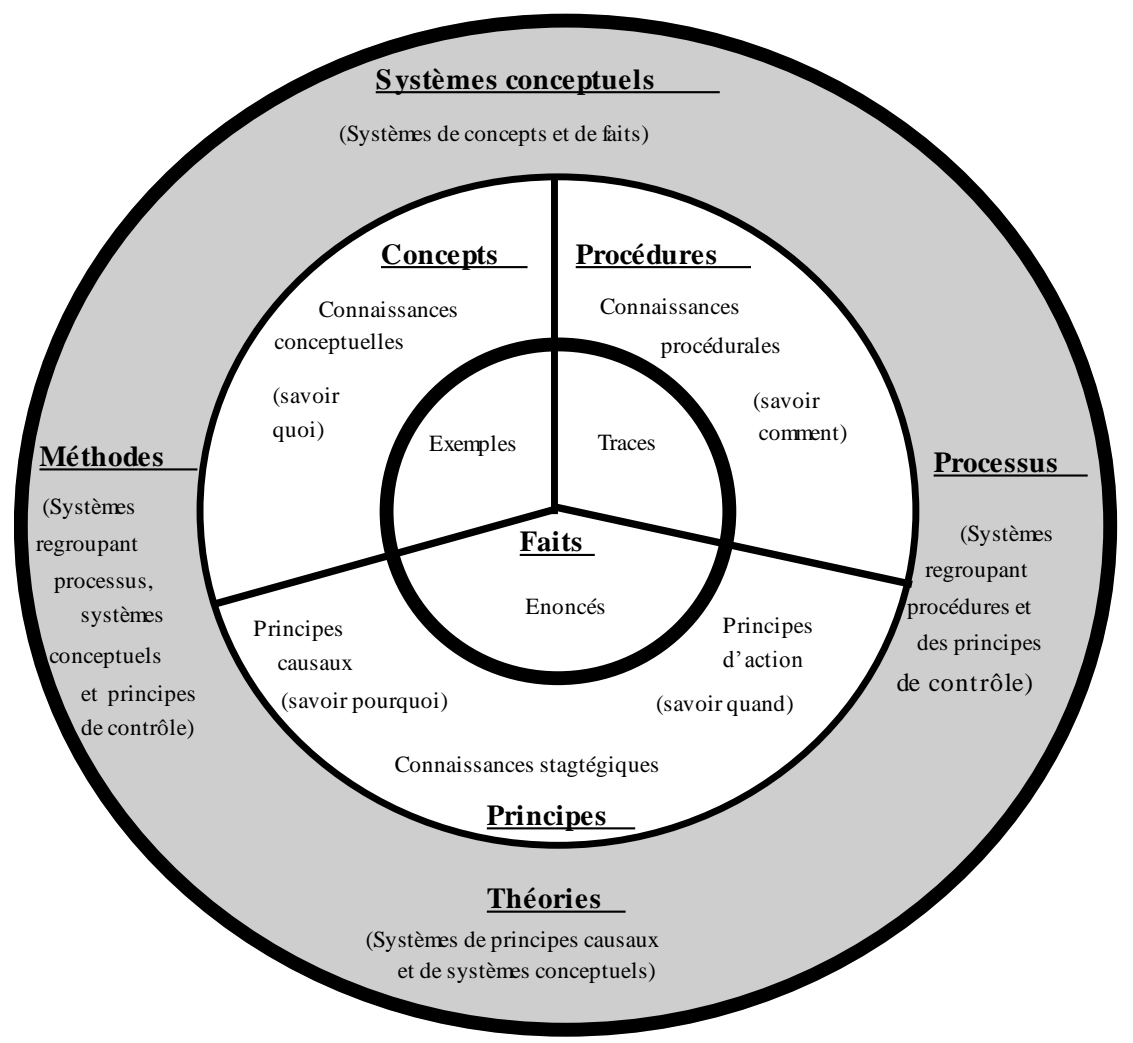

Figure 8 - Taxonomie des connaissances : faits et connaissances abstraites

Les théories sont des systèmes de connaissances regroupant plusieurs principes causaux (les lois ou les théorèmes) établissant des relations entre les termes (définitions) du domaine :

Exemple : en économie, la théorie de l'économie de marché regroupe notamment des lois comme celle de l'offre et de la demande, ainsi que les systèmes conceptuels définissant l'offre, la demande ou les échanges économiques.

Exemple : les lois et les concepts permettant d'expliquer la gravitation universelle

Les processus sont essentiellement des systèmes regroupant plusieurs procédures pouvant se dérouler séquentiellement ou en parallèle, lesquels sont déclenchées en fonction de connaissances stratégiques qui sont des règles de décision (déterministe) ou des règles heuristiques qui réglementent ces procédures. Un processus décrit également les intrants et les extrants des procédures qui le composent.

Exemple : le processus de planification pour la rédaction coopérative d'un texte ou celui permettant de construire un rapport d'impôt. 
Les méthodes sont des systèmes de plus grande envergure regroupant à la fois des processus, des principes méthodologiques ou des stratégies, ainsi que des systèmes conceptuels : réseaux de concepts, taxonomies, systèmes d'interprétation.

Exemple : la méthode de design pédagogique regroupe plusieurs processus tels que :

- l'analyse du problème de formation;

- la planification de la formation;

- la réalisation des instruments de formation;

- l'évaluation et la révision des instruments de formation;

- l'implantation et la gestion du système de formation.

\section{Formalisme de représentation MOT}

Dans cette section, nous utilisons le formalisme graphique développé dans la section précédente pour décrire les composantes du système de représentation MOT lui-même et identifier les règles d'intégrité du système.

C'est ce qu'on appelle construire un méta-modèle. Dans le présent méta-modèle, le système de représentation est décrit en tant que système conceptuel, c'est-à-dire que les objets du système MOT sont des concepts reliés par les relations de composition et de spécialisation. Autrement dit, le processus de modélisation, avec ses aspects procéduraux (comment modéliser) ou stratégiques (quand et pourquoi modéliser de telle ou telle façon) ne sera pas décrit ici.

\subsection{Concept du système de représentation MOT}

Dans la section précédente, nous avons présenté plusieurs exemples de modèles construits avec le système de représentation MOT. Ces modèles sont le résultat (l'extrant) du processus de modélisation. Nous décrivons d'abord ce qu'est un modèle MOT.

Un modèle MOT se compose d'une ou plusieurs unités de connaissance, d'un ou plusieurs liens et de contraintes d'intégrité que doivent respecter ces unités de connaissances et ces liens.

Tel que décrit à la figure 9 , une unité de connaissance possède :

—un et un seul nom (inscrit dans la case qui le représente graphiquement),

- un et un seul type : concept, procédure, principe ou instance d'un de ces trois types (représenté graphiquement par la forme de la case),

- un ou plusieurs attributs qui sont eux-mêmes des unités de connaissances, c'est-à-dire possèdant un nom, un type et un ou plusieurs attributs (à la limite, un attribut peut être un fait, c'est-à-dire prendre une valeur précise). 


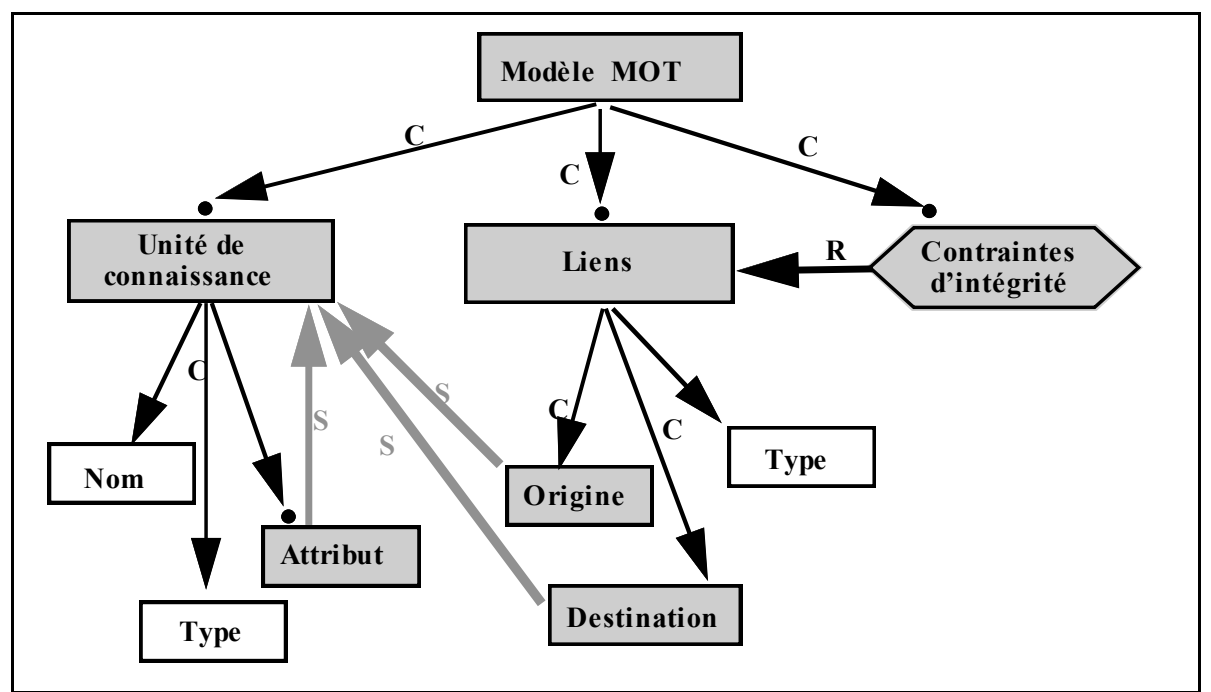

Figure 9 - Le concept de modèle dans le système de représentation MOT

Également, un lien possède:

—un et un seul type (inscrit sur la flèche qui le représente): I, C, S, P, I/E ou R,

-une origine qui est une unité de connaissance,

—une destination qui est aussi une unité de connaissance.

Les contraintes d'intégrité seront décrites à la section 3.4. En particulier, elles permettent ou interdisent certains liens selon les types des unités de connaissance qui sont à l'origine ou à la destination du lien.

\subsection{Types de connaissances et de modèles}

La figure 10 présente les différentes types d'unités de connaissance pouvant apparaître dans un modèle MOT, soit les systèmes de faits ou instances, les concepts, les principes et les procédures.

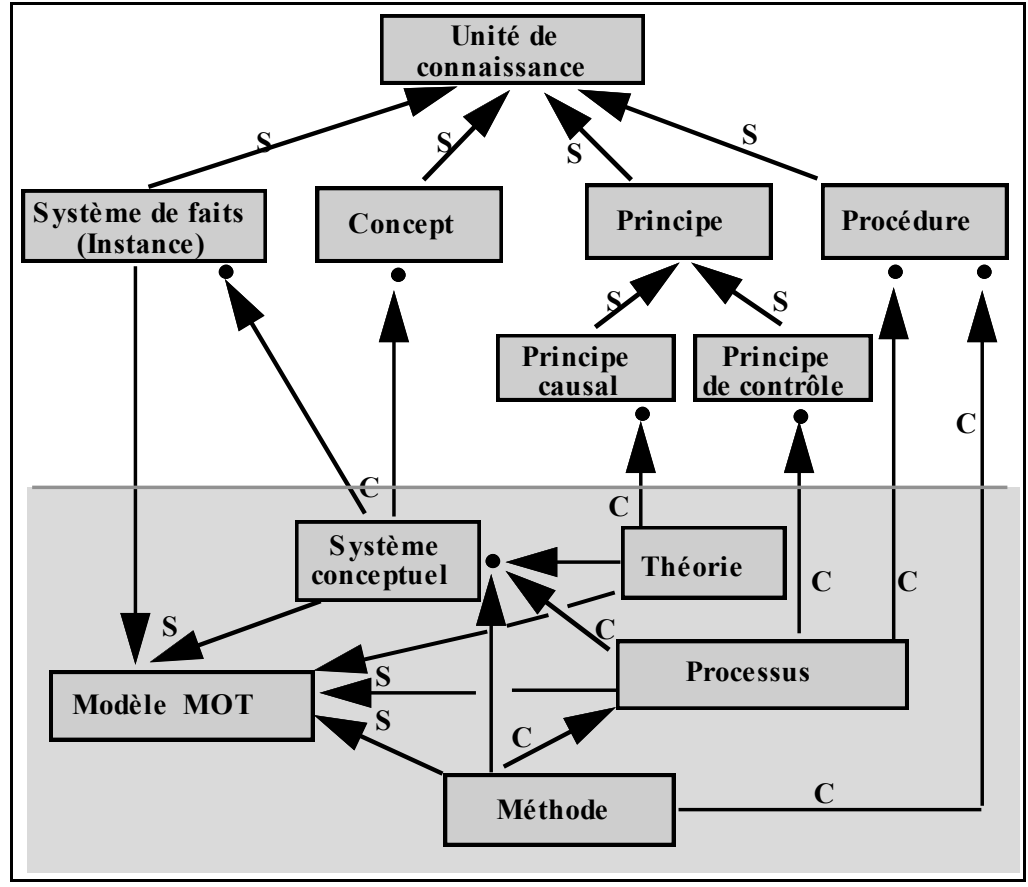

Figure 10 - Types de connaissances et de modèles MOT 
On distingue deux types de principes, les principes causaux et les principes de contrôle. Les premiers mettent en relation deux principes simples, une condition et une conclusion. Les seconds mettent en relation une condition et une ou plusieurs actions ou procédures.

Les systèmes de connaissances complexes présentés à la section 3.7 tels que les systèmes conceptuels, les processus, les méthodes et les théories, sont présentés ici comme des sortes de modèles plutôt que comme des unités de connaissances. Ces systèmes ne constituent pas de nouveaux types, mais se composent des types ou des sous-types de connaissances tel qu'illustré sur la figure 10:

-un système conceptuel est un modèle MOT qui se compose uniquement de concepts et de systèmes de faits;

—une théorie est un modèle MOT qui se compose de systèmes conceptuels et de principes causaux;

—un processus est un modèle MOT qui se compose de procédures, de systèmes conceptuels qui en sont les intrants ou les extrants et de principes de contrôle qui régissent l'exécution des procédures;

- une méthode est un modèle MOT qui se compose de processus, de systèmes conceptuels (généralement plus élaborés que dans un processus) et de principes de contrôle qui régissent l'exécution des processus.

\subsection{Types de liens}

Un lien est un objet de représentation composé d'un type (attribut interne), d'une origine et d'une destination. L'origine et la destination sont des sortes d'unités de connaissances : systèmes de faits, concepts, procédures ou principes.

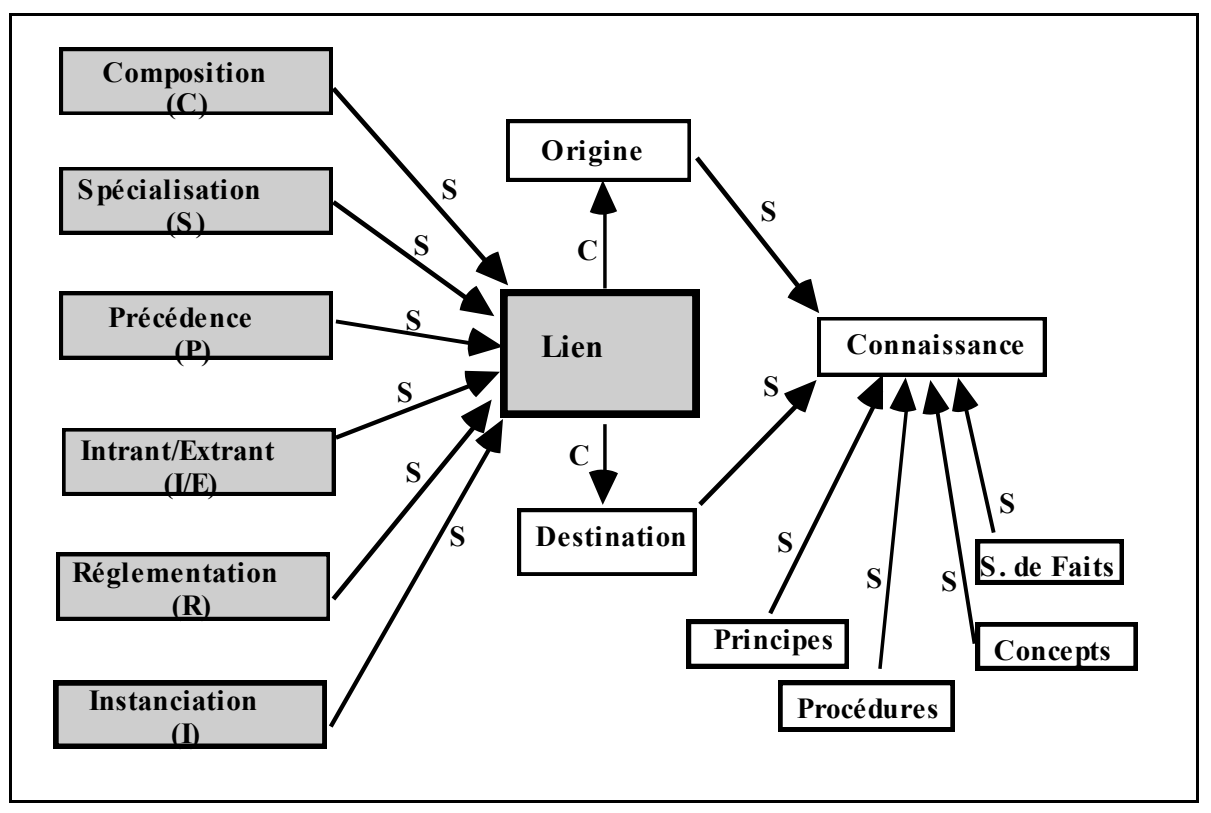

Figure 11 - Méta-modèle du concept de lien

Chacun des six types de liens illustrés sur la figure 11, est particulier en ce qui concerne son origine, sa destination et ses autres propriétés. Nous les passons brièvement en revue.

\section{Lien d'instanciation "a-pour-instance" (I)}

Le lien d'instanciation a nécessairement pour origine un concept, une procédure ou un principe et pour destination un système de faits. Les liens qui relient les faits dans une instance (et qui en font un système de faits), dépendent du type de connaissance abstraite qui leur a servi de moule : 
- Lorsqu'on instancie un concept, on donne une valeur concrète à tous les attributs, obtenant ainsi un exemple. Les faits qui constituent l'exemple sont liés en tant que propriétés possédées par un même individu.

- L'instanciation d'une procédure produit une trace d'exécution ; dans ce cas, les faits sont reliés entre eux par leur occurrence dans le temps lors de l'application de la procédure à un cas particulier.

-L'instanciation d'un principe donne lieu à un énoncé. Dans ce cas, s'il s'agit d'un principe causal, le lien entre les faits est de type causal entre les deux conditions instanciées. Dans le cas d'un principe de contrôle, le lien est entre la condition instanciée et la trace d'exécution de la partie action.

Exemple: $\quad$ Le principe causal "Si un triangle est rectangle, alors sa surface est le demi-produit des côtés bornant l'angle droit" s'intancie au système de faits suivant : "Le triangle $(90 \mathrm{o}, 60 \mathrm{o}, 30 \mathrm{o}$, $5,4,3)$ est rectangle, par conséquent sa surface et de $6 "$.

Exemple: Le principe de contrôle "Si un patient est fumeur et consomme graisses et alcool, alors il faut appliquer la procédure d'analyse des problèmes cardiaques" s'instancie au système de faits suivant: "M. Bureau est fumeur, il consomme graisses et alcool, et la procédure d'analyse des problèmes cardiaques a été appliquée et a permis le diagnostic suivant : pas d'infarctus, deux coronariennes bloquées, ....."

- L'instanciation d'un système conceptuel ou d'une théorie est un système de faits qui est souvent appelé modèle ou prototype. Les liens entre ces faits sont ceux qui permettent de décrire précisément les objets.

Exemple : En instanciant le système conceptuel qui décrit une automobile, on obtient un système de faits contenant des exemples des différentes parties de l'automobile : la carrosserie est bleue, la transmission est automatique, le moteur est de 6 cylindres,....; d'autres faits seront des traces telles que "la procédure d'entretien de la transmission passe par les vérifications a,b,c..."; d'autres faits résultant de l'instanciation seront des énoncés tels que "puisque la transmission avait un problème de friction l'huile a été remplacée".

- L'instanciation d'un processus ou d'une méthode est souvent appelée démarche de production d'un bien livrable ou d'un résultat, telle qu'on la consigne parfois dans un journal de bord.

Exemple: En appliquant la méthode de design pédagogique à un problème de formation des pilotes de Boeing 747, la procédure d'analyse a conduit à la décision de créer un système de formation sur mesure décrit dans le document 1.2.2; le processus de planification a permis de retenir la simulation interactive comme stratégie pédagogique ; le cours a ensuite été subdivisé en trois unités de formations ayant chacune les activités suivantes :.....; le processus de réalisation a permis de créer un logiciel de simulation, deux audio-visuels de 30 minutes et un document d'accompagnement regroupant les activités de formation.

\section{Lien de composition "se-compose-de" (C)}

Il y quatre cas possibles selon l'origine du lien :

Si l'origine est un fait, la destination est nécessairement un fait ; un fait (ou plutôt un système de faits) ne peut se composer de concepts, de procédures ou de principes qui sont des connaissances plus abstraites;

Si l'origine est un concept, la destination (un de ses attributs) peut être un fait, un autre concept, une procédure ou un principe ; les figures 1 et 3 de la section 2 présentent des exemples de ces trois derniers cas ;

— dans le cas où un des attributs du concept a une valeur précise, celui-ci a pour composante un fait.

Exemple : le "Nb enfants est 0" est une composante du concept de "famille sans enfant";

- les destinations de type "procédure" sont des "attachements procéduraux" qui permettent d'assigner à un attribut le résultat d'une procédure dépendant d'autres attributs du concept. 
—les destinations de type "principe" sont des "contraintes d'intégrité" qui restreignent le nombre d'instances possibles, celles-ci devant respecter la contrainte.

Exemple : une des angles doit mesure 90o, composante du concept de "triangle rectangle".

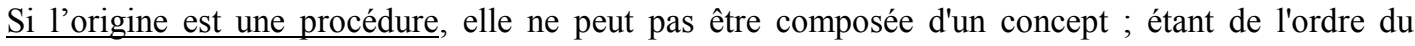
"comment", elle consiste à décrire comment faire des choses et ne peut avoir comme composante que d'autres procédures ou des principes de contrôle (ou règles de décision).

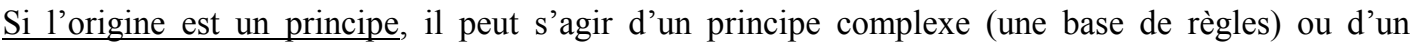
principe simple de type causal ou de type contrôle

- une base de règles (par exemple pour le choix d'un médicament ou pour classifier un objet) sera considérée comme un principe complexe qui se décompose en principes plus élémentaires ;

- les principes causaux et les règles d'intégrité peuvent mettre en relation deux composantes qui sont elles-mêmes des énoncés de principes ;

Exemple : Si le taux d'alcool dans le sang est anormal, alors l'ivressomètre indiquera une valeur égale ou supérieure à 8 .

Exemple : Si une phrase contient le verbe avoir et si le complément d'objet direct est placé avant le verbe, alors le participe passé s'accorde en genre et en nombre avec le complément d'objet direct.

- les principes de contrôle ont une composante "Si" qui est une condition, donc un principe, et une composante "Alors" qui est une procédure;

Exemple: Si une figure géométrique est complexe et que l'on désire y découvrir des relations métriques, alors il est utile de trianguler la figure".

Exemple : Si le contribuable est propriétaire, alors il faut remplir l'annexe $\mathrm{J}$ du rapport d'impôt sur le revenu.

Lien de spécialisation "est-sorte-de" (S)

La relation de spécialisation "est-sorte-de" ne peut s'établir qu'entre connaissances abstraites de même type. Elle ne peut avoir comme origine ou destination un fait. Autrement dit, deux connaissances ne peuvent être liées à la fois par un lien I, et un lien $\mathrm{S}$. De plus, deux connaissances ne peuvent être liées à la fois par un lien $\mathrm{C}$ et un lien S. On peut faire ici l'analogie avec les relations ensemblistes : si un objet est élément (composante) d'un ensemble, il ne peut en même temps être un sous-ensemble (sorte-de) de cet ensemble.

Exemples:

—Les mammifères sont une sorte d'animaux vertébrés.

- La procédure de tri par ordre alphabétique est une sorte de tri.

—-Deux triangles sont égaux si leurs côtés sont égaux" est une sorte de principe d'égalité des triangles.

$\underline{\text { Lien de précédence }(\mathrm{P})}$

La figure 5 de la section 2 illustre les liens de précédence entre les sous-procédures et les règles de décision qui composent un algorithme récursif. La relation de précédence n'a de sens qu'entre des procédures ou des principes de contrôle (règles de décision).

\section{Lien intrant ou extrant (I/E)}

La relation intrant/extrant est une forme de précédence, mais réservée aux liens entre concepts (ou instances de concepts) et procédures. Une procédure consiste essentiellement à traiter des données qui sont des instances d'un concept et inversement à produire de nouvelles données qui sont également des instances de concept. Dans le premier cas, on dira qu'il y a un lien I/E du concept ou de l'instance de concept vers la procédure et, dans le deuxième cas, qu'il y a un lien $\mathrm{I} / \mathrm{E}$ de la procédure vers le concept ou l'instance de concept.

Les figures 5, 6 et 7 nous ont permis d'illustrer ce type de lien. Ainsi, les concepts de plan, de brouillon ou de version finale d'un texte servent d'intrant/extrant aux procédures de rédaction d'un texte. 
Un lien intrant/extrant entre deux connaissances exclut tout autre type de lien,

- un fait (instance de concept) ou un concept intrant à une procédure ne peut être une instance de celle-ci puisque que ce n'est pas une trace de procédure ; il en est de même des extrants;

- le lien de précédence ne se place qu'entre procédures ou règles de décision;

- une procédure ne peut se composer de concepts ou de faits, ni être une sorte de concept ou de faits.

- un concept ne peut se composer d'une procédure et en être l'intrant ou l'extrant puisqu'il aurait besoin de cette procédure pour lui fournir des valeurs.

\section{$\underline{\text { Lien de réglementation }(\mathrm{R})}$}

Le lien de réglementation est réservé aux principes lorsque, de l'extérieur, ils contraignent, réglementent ou régissent une procédure ou un concept. Ce lien a donc toujours comme origine un principe et comme destination une procédure ou un concept.

Si la destination est une procédure, le principe est nécessairement une base de règles d'action, lesquelles contrôlent l'exécution de la procédure. Les règles causales n'ont pas de composante "action" et ne peuvent donc jouer ce rôle.

\section{Exemples}

- Les normes de présentation régissant la production de certains types de documents.

—Les règles pédagogiques régissant la formulation des objectifs d'un cours.

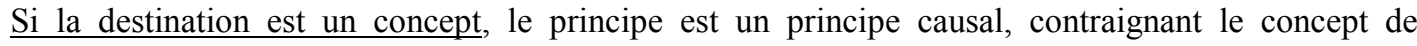
l'extérieur et régissant ainsi la façon dont il pourra être spécialisé ou instancié.

Exemples:

—-Les règles de grammaire qui définissent indirectement le concept de texte rédigé en langue française ;

—Les lois de la cinématique définissent indirectement le concept de mouvement d'un corps.

\subsection{Contraintes d'intégrité}

Nous présentons dans ce paragraphe une synthèse du modèle et de ses règles d'intégrité qui découlent du tour d'horizon qui précède. Le tableau 1 résume les liens possibles entre les quatre types de connaissances. 


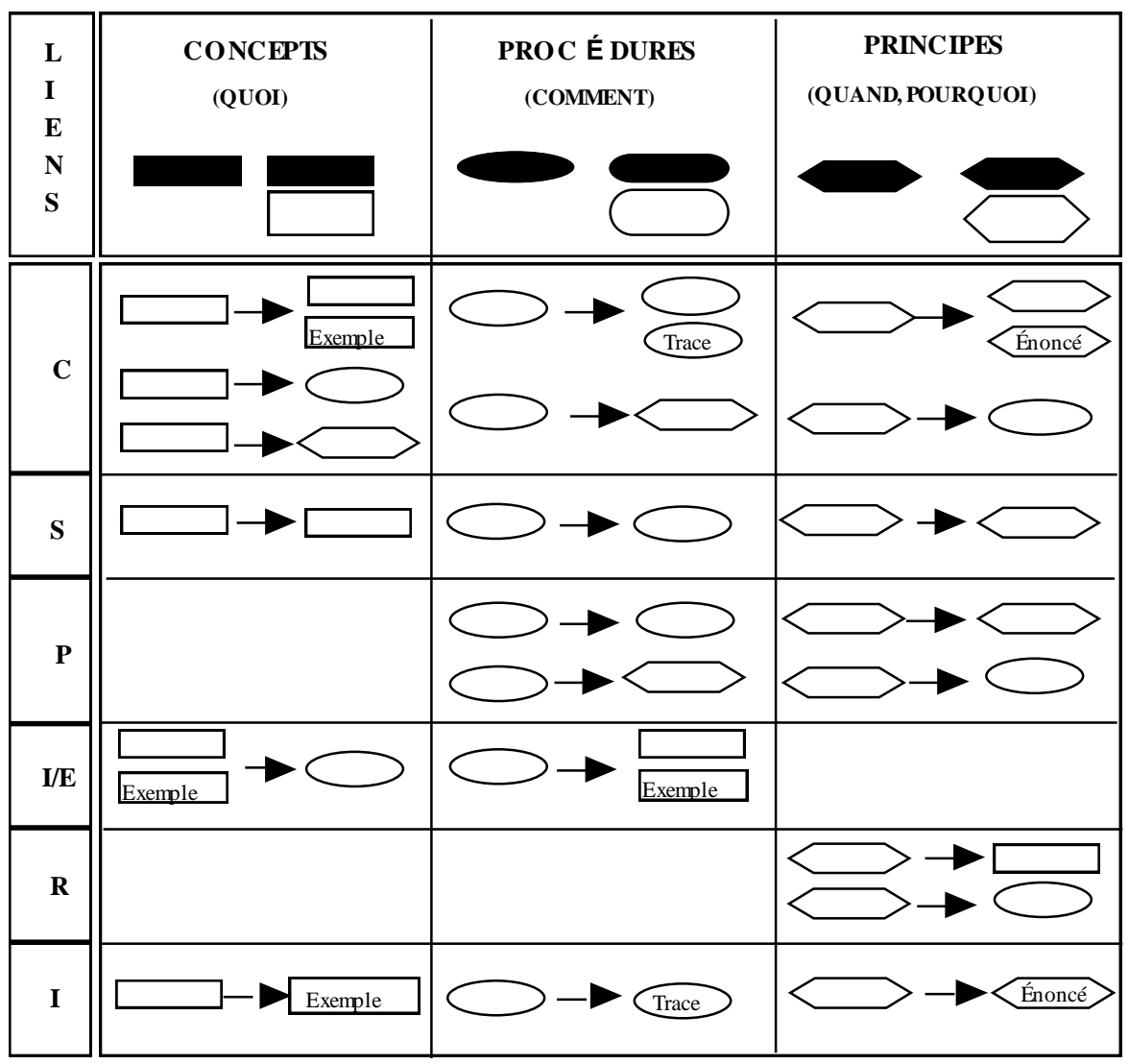

Tableau 1 - Synthèse du symbolisme graphique du modèle

En résumé,

-Une instance peut être une instance d'un concept, d'une procédure ou d'un principe. Elle peut aussi être intrant ou extrant d'une procédure, mais elle n'est impliquée dans aucun autre type de lien.

-Un concept peut être une sorte de concept, peut se composer d'autres concepts, de procédures ou de principes ; il peut être intrant ou extrant d'une procédure.

-Une procédure peut être une sorte de procédure, se composer de procédures ou de principes de contrôle, ou encore précéder une autre procédure ou un principe.

-Un principe peut être une sorte de principe, peut régir une procédure ou un concept, se composer d'autres principes ou de procédures ou précéder (ou suivre) une procédure.

Nous complétons la présentation du système de représentation par un énoncé des contraintes d'intégrité des modèles, résumées dans le tableau 2

\begin{tabular}{|l|c|c|c|c|c|c|}
\hline RÈGLE D'INTÉGRITÉ DES LIENS & $\begin{array}{c}\text { Lien } \\
\text { C }\end{array}$ & $\begin{array}{c}\text { Lien } \\
\text { S }\end{array}$ & $\begin{array}{c}\text { Lien } \\
\text { P }\end{array}$ & $\begin{array}{c}\text { Lien } \\
\text { I/E }\end{array}$ & $\begin{array}{c}\text { Lien } \\
\text { R }\end{array}$ & Lien I \\
\hline $\begin{array}{l}\text { Anti-réflexivité Pour toute } \\
\text { connaissance A, A n'est pas lié à A }\end{array}$ & $\sqrt{ }$ & $\sqrt{ }$ & $\sqrt{ }$ & $\sqrt{ }$ & $\sqrt{ }$ & $\sqrt{ }$ \\
\hline $\begin{array}{l}\text { Asymétrie Pour toutes } \\
\text { connaissances A et B, si A est lié à B, B } \\
\text { n'est pas lié à A }\end{array}$ & $\sqrt{ }$ & $\sqrt{ }$ & $\sqrt{ }$ & $\sqrt{ }$ & $\sqrt{ }$ & $\sqrt{ }$ \\
\hline
\end{tabular}




\begin{tabular}{|c|c|c|c|c|c|c|}
\hline $\begin{array}{l}\text { Transitivité } \quad \text { Pour toutes } \\
\text { connaissances } \mathrm{A}, \mathrm{B} \text { et } \mathrm{C} \text { si } \mathrm{A} \text { est lié à } \mathrm{B} \\
\text { et } \mathrm{B} \text { est lié à } \mathrm{C} \text {, alors } \mathrm{A} \text { est lié à } \mathrm{C}\end{array}$ & $\sqrt{ }$ & $\sqrt{ }$ & $\sqrt{ }$ & $\mathrm{n} / \mathrm{a}(4)$ & $\mathrm{n} / \mathrm{a}(4)$ & $\mathrm{n} / \mathrm{a}(4)$ \\
\hline $\begin{array}{l}\text { Unicité du lien } \\
\text { connaissance A et B, si A est lié à B, A } \\
\text { n'a aucun autre type de lien avec B }\end{array}$ & $\sqrt{ }$ & $\sqrt{ }$ & $\sqrt{ }$ & $\sqrt{ }$ & $\sqrt{ }$ & $\sqrt{ }$ \\
\hline $\begin{array}{l}\text { Typage de l'origine du lien Il n'y a } \\
\text { pas de restriction quant au type de } \\
\text { l'origine du lien }\end{array}$ & $\sqrt{ }$ & $\begin{array}{c}\text { Pas un } \\
\text { fait }\end{array}$ & $\begin{array}{l}\text { Procédur } \\
\text { e ou } \\
\text { principe }\end{array}$ & $\begin{array}{l}\text { Pas un } \\
\text { principe }\end{array}$ & Principe & $\begin{array}{c}\text { Pas un } \\
\text { fait }\end{array}$ \\
\hline $\begin{array}{l}\text { Typage de la destination du lien Il n'y } \\
\text { a pas de restriction quant au type de la } \\
\text { destination du lien }\end{array}$ & $\begin{array}{l}\sqrt{ } \\
(1)\end{array}$ & $\begin{array}{l}\text { Pas un } \\
\text { fait (2) }\end{array}$ & $\begin{array}{l}\text { Procédur } \\
\text { e ou } \\
\text { principe }\end{array}$ & $\begin{array}{l}\text { Pas un } \\
\text { principe } \\
(3)\end{array}$ & $\begin{array}{l}\text { Concept } \\
\text { ou } \\
\text { procédure }\end{array}$ & Fait \\
\hline $\begin{array}{l}\text { Cardinalité du lien Une connaissance } \\
\text { peut avoir } 0 \text {, un ou plusieurs liens }\end{array}$ & $\sqrt{ }$ & $\sqrt{ }$ & $\sqrt{ }$ & $\sqrt{ }$ & $\sqrt{ }$ & $\sqrt{ }$ \\
\hline $\begin{array}{l}\text { Unicité du type de destination Si une } \\
\text { connaissance a plus d'une destination, } \\
\text { celles-ci sont de même type. }\end{array}$ & Faux & $\sqrt{ }$ & Faux & $\sqrt{ }$ & Faux & $\sqrt{ }$ \\
\hline
\end{tabular}

(1) Une procédure ou un principe ne peuvent avoir comme composante un concept.

(2) La connaissance de destination d'un lien $\mathrm{S}$ doit être du même type que l'origine du lien.

(3) Si l'origine est un concept ou un fait, la destination d'un lien I/E doit être une procédure ; si l'origine est une procédure, la destination doit être un concept ou un fait.

(4) La question de transitivité ne se pose pas, car si A régit B, B est un concept ou une procédure et ne peut régir $\mathrm{C}$; si $\mathrm{A}$ est intrant-extrant à $\mathrm{B}$, et $\mathrm{B}$ à $\mathrm{C}, \mathrm{A}$ et $\mathrm{C}$ sont deux concepts ou exemples qui ne peuvent être intrant-extrant l'un de l'autre ; si A a pour instance B, B ne peut être instancié.

\section{Tableau 2 - Règles d'intégrité des modèles de connaissance}

Les relations de spécialisation $(\mathrm{S})$, de composition $(\mathrm{C})$ et de précédence $(\mathrm{P})$ sont des relations d'ordre partiel :

—elles sont antiréflexives : une connaissance ne peut être liée à elle-même;

—elles sont asymétriques : si une connaissance est liée à une autre, la réciproque n'est pas admissible;

- elles sont transitives : si $\mathrm{A}$ est lié à $\mathrm{B}$ et $\mathrm{B}$ à $\mathrm{C}$ par un de ces liens, $\mathrm{A}$ est lié à $\mathrm{C}$ (ces liens de transitivité étant généralement sous-entendus et non indiqué sur les modèles);

- elles ne sont pas totales, c'est-à-dire qu'il est possible que ni $\mathrm{A}$ ne soit liée à $\mathrm{B}$, ni B à $\mathrm{A}$.

Les relations d'intrant/extrant (I/E), de réglementation $(\mathrm{R})$ et d'instanciation (I) ne sont pas des relations d'ordre, car elles ne sont pas transitives.

Pour les six types de lien

- s'il y a un lien entre deux connaissances, il ne peut y avoir d'autre type de lien;

- dépendant du type de lien, il y a des restrictions quant au type de connaissances servant d'origine ou de destination du lien tel qu'indiqué sur le tableau 1;

- pour tous les types de liens, une connaissance peut être liée a aucune, une ou plusieurs destinations ;

- s'il y en a plus d'une destination pour un lien S, I/E ou I, celles-ci sont de même type, ce qui n'est pas le cas des autres liens.

\section{Modélisateur graphique}


Le système de modélisation présenté ici a dépassé le stade théorique, bien qu'il continue à faire l'objet de recherche. Un outil informatisé de modélisation a été réalisé et mis à l'essai auprès d'usagers. Ce modélisateur graphique permet de construire de façon interactive un modèle de connaissance composé de connaissances et de liens typés, respectant les contraintes d'intégrité qui régissent celles-ci dans le système de représentation MOT.

Figure 12 - L'éditeur de modèles de connaissances par objets typés

La figure 12 est une recopie d'écran de l'interface qui s'offre à l'usager :

- le panneau central permet de disposer, sélectionner et déplacer les unités de connaissances et les liens qui composent un modèle par objets typés ;

- une fenêtre annexe peut être affichée par un double-clic sur une connaissance, permettant de lui attribuer un nom ; le champ commentaire peut servir à décrire les attributs internes de cette connaissance ;

- une autre fenêtre annexe peut être affichée, présentant une structure hiérarchique dans laquelle on peut répartir le modèle de connaissance ; il suffit de sélectionner un ensemble d'unités de connaissances et de les "glisser-déposer" sur un des icônes de la hiérarchie pour créer un sousmodèle qui conserve les liens du modèle original ;

- le même type d'opération permet de transférer des connaissances (et leurs liens) d'un sousmodèle à un autre ou du modèle d'un projet à celui d'un autre projet ;

- les barres de menus et de boutons permettent de créer, effacer localement, détruire les unités de connaissances ou les liens ; de faire diverses opérations de zoom sur le modèle ; de disposer les fenêtres ; d'afficher la hiérarchie des sous-modèles ; de créer, sauver, fermer ou détruire un projet de modèle et de l'imprimer ; éventuellement des opérations de filtres par type de connaissance, par type de lien ou selon la proximité topologique, déjà implantées dans une version antérieure, seront intégrées à l'éditeur pour faciliter l'analyse et le développement des modèles.

De plus, les contraintes d'intégrité présentées à la fin de la section 3 ont été implantées sous la forme de messages. Si l'usager décide de tracer un lien entre deux connaissances dont le type n'est pas compatible avec celles-ci, le modélisateur indiquera quelle contrainte n'est pas respectée et suggérera d'autres liens possibles entre les connaissances visées.

\section{Discussion des résultats}

Le modélisateur graphique, a été mis à l'essai, dans le cadre du développement et de la validation de l'atelier de génie didactique ${ }^{11}$ dont nous avons parlé plus haut, ainsi qu'à l'extérieur de ce cadre :

- un certain nombre d'usagers du groupe DMR ${ }^{12}$ l'ont utilisé au cours de son développement (en 1994) et ensuite, dans le cadre de la réalisation d'un cours d'introduction à la méthode $\mathrm{P}+$ développée par cette entreprise (depuis juin 1995) ;

-l'auteur a utilisé le formalisme lors de la définition conceptuelle du système d'aide à la

performance que constitue l'atelier de génie didactique (mai-août 1994);

- une session de dix jours à la Banque de Montréal a permis aux quatre concepteurs impliqués de réaliser le devis d'un curriculum à l'intention des conseillers aux prêts (mars 1995) ;

\footnotetext{
${ }^{11} \mathrm{~L}$ 'auteur tient ici à remercier l'équipe du projet AGD, particulièrement Eric Bleisher qui a fait l'analyse et la réalisation de l'outil de modélisation, ainsi que Claire Aubin et Françoise Crevier qui ont contribué à sa mise au point en fonction des impératifs de la conception pédagogique, tout en assumant la responsabilité de la validation de l'AGD.

12 Le groupe DMR, importante compagnie de consultation en informatique, a été à l'origine du projet AGD, dans le cadre duquel nous avons réalisé l'outil de modélisation présenté ici.
} 
-le modélisateur a servi à la définition du contenu de deux cours de la Télé-université, l'un sur la résolution de problème (avril-mai 1995) et l'autre en linguistique (depuis juin 1995);

- lors d'une session d'évaluation intensive, un expert dans le domaine du design automatisé, a développé un contenu de formation dans le domaine de la défense (mai 1995) ;

—dans le cadre d'un projet de modélisation de l'informatique à l'école, la technique de modélisation sert à définir les processus et les acteurs des sous-systèmes d'apprentissage en classe, d'information et de communication, de gestion pédagogique et administrative (depuis juin 1995).

Les résultats obtenus jusqu'à maintenant, bien que partiels ${ }^{13}$, sont prometteurs en regard des objectifs que nous nous étions fixés, en particulier la simplicité d'utilisation par des non initiés, l'expressivité du formalisme dans une variété de situations et de domaine de connaissance, et la transparence des représentations du point de vue sémantique.

Dans l'ensemble, les usagers ont maîtrisé l'outil et la technique de modélisation en quelques heures d'apprentissage, réussissant à construire des modèles représentatifs de différents domaines de connaissances. Certains concepteurs pédagogiques ${ }^{14}$ ont affirmé que le système leur avait permis de réduire considérablement le temps consacré à la définition du contenu de l'apprentissage et à sa répartition dans les cours, les modules et les activités d'apprentissage. D'autres ont souligné la convivialité et la pertinence de l'approche de modélisation utilisée ${ }^{15}$.

Lorsque la validation de la technique de modélisation sera complétée, nous prévoyons une extension des fonctionnalités de l'outil de modélisation appuyée par des recherches sur les questions suivantes :

—Extension du modèle pour couvrir le domaine des métaconnaissances et des habiletés cognitives;

—Adaptation du modèle pour couvrir plus complètement la représentation de la collaboration et la coopération dans les processus multi-agents;

- Intégration de la modélisation à la formulation d'une méthodologie de développement de systèmes informatisés de support à la performance et d'apprentissage.

\section{Bibliographie}

[Anderson 1985] J. R. Anderson. Cognitive psychology and its implications. New York, Freeman.

[Bartlett 1932] F.C. Bartlett. Remembering. Cambridge University Press, England.

[Bond et Gasser 1988] A. H. Bond et L. Gasser editors. Readings in Distributed Artificial Intelligence. Morgan Kaufmann, San Mateo, California.

[Ferber, 1994] J. Ferber. Coopération réactive et émergence, Revue Intellectica, No2, pp. 19-52

[Gasser, 1991] L. Gasser. Social conceptions of knowledge and action: DAI foundations and open system semantics. Artificial Intelligence, 47: 107-138.

[Bruner 1973] J. Bruner. Beyond the information given. New York, Norton.

13 Une validation scientifique est en cours auprès de quatre organismes

14 David Peach, Institute for Learning, Bank of Montreal

15 Mike Spector, Armstrong Laboratory, U. S. Air Force [Spector et al, 1993] 
[Chen, 1976] P.P.S Chen. The Entity-Relationship model - toward a unified view of data. $A C M$ Transactions on Database Systems I,1 (march 1976)

[Inhelder et Pidaget 1958] B. Inhelder \& J. Piaget. The growth of logical thinking from childhood to adolescence. New York: Basic Books.

[Merrill 1994] D. Merrill. Principles of Instructionnal Design. Educationnal Technology Publications, Englewood Cliffs, New Jersey, 465 pages.

[Minski 1975] M. Minski. A framerwork for reprsenting knowledge. In P. H. Winston (ED.), THe psychology of computer vision. New York: McGraw-Hill.

[Newell et Simon 1972] S. Newell \& H. Simon. Human problem solving. Englewood Cliffs, NF: Prentice-Hall.

[Paquette, 1993] G. Paquette. Un système de modélisation cognitive et métacognitive. pour l'ingénierie didactique Notes de recherches du LICEF, Novembre 1993.

[Paquette et al, 1994] G. Paquette, F.Crevier et C. Aubin ID Knowledge in a Course Design Workbench. Educationnal Technology, USA, volume 34, n. 9, pp. 50-57, November 1994.

[Paquette et al, 1995] G. Paquette , C. Ricciardi-Rigault, J. Bourdeau, C. Paquin \& S. Liégeois. Modeling a Virtual Campus Environment for Interactive Distance Learning, ED-Media International Conference, Graatz, Austria, juin 1995.

[Paris et al, 1983] S. Paris, M.Y. Lipson, \& K.K. Wixson. Becoming a strategic reader. Contemporary Educational Psychology, 8, 293-316.

[Romiszowski 1981] A. J. Romiszowski. Designing Instructional Systems. Kogan Page London/Nichols Publising, New York, 415 pages.

[Rumbaugh et al, 1991] J. Rumbaugh, M. Blaha, W. Premerlani, F. Eddy, W Lorensen. ObjectOriented Modelling and Design. Prentice Hall, Englewood Cliffs, New Jersey.

[Rumelhart et Ortony.1977] D.E. Rumelhart \& A. Ortony. The representaion of knowledge in memory. in R. C. Anderson, R.J. Spiro \& W. E. Montague (Eds) Schooling and the acquisition of knowledge. Lawrence Erlbaum. Hillsdale, NJ.

[Scheiber et al, 1993] G. Schreiber, B. Wielinga, J. Breuker. KADS: A Principled Approach to Knowledge-Based System Development. Academic Press, London.

[Spector et al, 1993] J.M. Spector, M.C. Polson, D.J. Muraida (Eds) Automating Instructional Design, Concepts and Issues, Educational Technology Publications, Englewood Cliffs, New Jersey, 364 pages.

[Sowa, 1984] J.F. Sowa. Conceptual Structures, Information Processing in Mind and Machine, Addison-Wesley Pubilishing Co, Reading, Mass, 481 pages.

[Tardif, 1992] J. Tardif. Pour un enseignement Stratégique, l'apport de la psychologie cognitive. Les éditions LOGIQUES, Montréal.

[Tennyson et Rasch, 1998] R. Tennyson. Linking cognitive learning theory to instructional prescriptions. Instructional Science, 17, pp. 369-385.

[Wertheimer 1945] M. Wertheimer. Productive thinking. New York: Harper \& Row. 
[West, 1991] C. K. West, J. A. Farmer, P. M. Wolff. Instructional Design, Implications from Cognitive Science. Allyn and Bacon, Boston, 271 pages.

[Winograd 1985] T. Winograd. Beyond the declarative/procedural controversy, in R. J. Brachman \& H. J. Lévesque (Eds), Readings in Knowledge Representation. Morgan Kaufmann Publishers.

[Yourdon, 1989] E. Yourdon. Modern Structured Analysis. Prentice Hall, Englewood Cliffs, New Jersey.

\section{Notice biographique}

Gilbert Paquette, est professeur à la Télé-Université à Montréal où il dirige le Laboratoire de recherche en informatique cognitive et environnements de formation (LICEF). Après des études de maîtrise et de doctorat à l'Université de Montréal, il a obtenu un doctorat en informatique de l'Université du Maine : "Métaconnaissances dans les environnements d'apprentissage". Pionnier du mouvement LOGO au Québec, il a animé les projets LOUPE et LOUTI en IA et éducation et, plus récemment, les projets CAMPUS VIRTUEL portant sur les méthodes et les outils de télé-apprentissage, AGD (Atelier de Génie didactique), un système d'aide à la conception de système d'apprentissage et ÉPITALK, un système de support à la réalisation de systèmes conseillers intelligents. 